Reprod. Nutr. Dévelop. 1980, 20 (2), 381-418.

\title{
Les hormones gonadotropes de l'hypophyse : biochimie et biologie comparées; spécificité et évolution
}

\author{
par Y. A. FONTAINE
}

\author{
Laboratoire de Physiologie générole ef comparée du Muséum \\ national d'Histoire nafurelle. Laboratoire d'Endocrinologie \\ comparée associé au C.N.R.S. \\ 7, rue Cuvier, 75231 Paris cedex 05.
}

Summary. The pituitary gonadotropic hormones : comparative biochemistry and biology ; specificity and evolution.

This review summarizes recent data on the nature of pituitary gonadotropins (GTH) and their biosynthesis, secretion, control and biological effects in vertebrates.

While the existence of $\mathrm{LH}$ - and FSH-like hormones has been demonstrated in a number of tetrapods, it is still controversial in fish. With the possible exception of the stimulation of yolk protein uptake in oocytes, one GTH may assume the various gonadotropic functions of the pituitary. Like mammalian GTH's, the fish GTH's purified so far have two subunits. Using carp gonadotropin (c-GTH), the partial sequences of these subunits were determined to be homologous to the mammalian $\alpha$ and $\beta$ types. c-GTH $\beta$ was also shown to be closer to mammalian $\mathrm{LH} \beta$ than to $\mathrm{FSH} \beta$. Immunological studies have confirmed these findings and demonstrated that, from fish to mammals, the antigenic determinants were better conserved for LH $\beta$ than for the $\alpha$ subunits. An hybrid molecule, formed by associating bovine $\mathrm{LH} \alpha$ and $\mathrm{c}-\mathrm{GTH} \beta$, was more active in frogs than either of the mother-hormones, while it was inactive on adenylate cyclase in homogenates of rat or goldfish ovaries. This hybrid, however, was as active as c-GTH in increasing c-AMP concentration in pieces of eel ovary. Interestingly, sturgeon gonadotropin (aci-GTH) had somewhat similar biological properties. Aci-GTH subunits were partially purified, and an hybrid (bovine LH $\alpha$-aciGTH $\beta$ ) was created. Both of the possible hybrids from the c-GTH and aci-GTH subunits could also be formed.

The kinetics of subunit association have been studied in vitro. As compared with the formation of ovine LH, that of c-GTH was more rapid and less thermodependent. Thus, even though fish may live at low temperatures, GTH synthesis proceeds at a reasonable rate. The thermodependence of the formation of the hybrid, bovine LH $\alpha-c-G T H \beta$, was identical to that of the c-GTH. Therefore, the $\beta$ subunit alone seems to determine thermodependence.

The data on specific gonadal response to GTH are reviewed in fish, amphibians, reptiles, birds and mammals. The physiological specificity of endogenous GTH or GTH's has been compared to their zoological specificity among vertebrates. In mammals, for example, c-GTH was inactive on ovarian adenylate cyclase in 16 to 18-day old rats in vitro, but did stimulate that enzyme in 20 to 24-day old rats (It had been shown previousiy that the physiological receptivity of receptors also changed at 19 to 20 days post-partumn).

All the data have been discussed from an evolutionary viewpoint in an attempt to define a « physiology of evolution ». The various GTH subunits in living vertebrates probably derive from a common ancestral molecule. This complex phylogenesis includes modifications at functional sites like those governing $\alpha$ and $\beta$ association and cell hormone 
binding. Several hypotheses have been proposed for this molecular evolution. The $\beta$ subunit would play a major role in the determinism of functional and zoological specificities of action, i.e. hormene recognition by the target cells.

The cellular structures recognizing the hormones and permitting them to act also evidence modifications during vertebrate evolution. It is difficult to speculate on these changes since the exact nature of the receptors is unknown. However, it is suggested that each strategy (hormenal change, receptor modification) prevailed at different stages of vertebrate phylogenesis.

Some concepts in comparative biology, such as the idea of receptor variability, are discussed : (i) even within one species, the specificity of the different receptors for the same hormone may vary (ii) there are probably several types of receptors with different specificities (iii) specificity in a same type may also vary with the physiological conditions.

It is finally pointed out that some species of lower vertebrates may be useful experimental models for studying general problems in reproductive biology.

\section{Abréviations}

GTH gonadotropine.

LH lutropine.

FSH follitropine.

TSH thyréotropine.

CG gonodotropine chorionique.

La ou les lettres minuscules placées avant les sigles des hormones désignent l'origine zoologique de ces dernières (initiale ou initiales du nom latin du genre) : c (de carpe), aci (d'esturgeon), het (de poisson-chat indien), onc (de saumon du Pacifique), ang (d'anguille), ti (te Tilapia), scy (de roussette), h (d'homme), o (de mouton), b (de bœuf), su (de porc).

PMSG gonadotropine de sérum de jument gravide.

LHRH hormone hypothalamique de Mammifères entraînant une décharge de LH et FSH.

AMPc $\quad 35^{\prime}$ adénosine monophosphate cyclique.

GMPc 3'5' guanosine monophosphate cyclique.

AC adénylate cyclase.

PK protéine kinase.

PAGE électrophorèse en gel de polyacrylamide.

$K_{D} \quad$ coefficient d'exclusion en filtration sur gel.

AS antisérum.

RIA dosage radioimmunologique.

La biologie comparée des hormones gonadotropes hypophysaires constitue, pour plusieurs raisons, un thème de recherche en plein développement. La diversité des Vertébrés, liée à leur phylogénie et aux adaptations à des environnements très variés, se reflète dans les modalités du contrôle des gonades ; en plus de leur intérêt fondamental, les recherches en physiologie comparée de la reproduction sont donc susceptibles de fournir des modèles originaux utiles pour la solution de problèmes de biologie générale. D'un autre côté, la connaissance des propriétés des GTH de certaines espèces dont l'élevage présente un intérêt économique est nécessaire : elle permettrait une application fructueuse des traitements hypophysaires qui maintiennent ou accélèrent la reproduction, souvent difficile à obtenir chez des animaux en captivité. Un troisième aspect, enfin, concerne l'évolution biochimique et physiologique. Une circonstance où ce thème peut être abordé, avec prudence certes, mais avec quelques chances 
de succès au moins partiel, est celle où des protéines fonctionnelles homologues sont mises en jeu, l'homologie désignant leur propriété d'être synthétisées grâce à des gènes dérivant d'un gène ancestral commun (Florkin, 1966). Or, de nombreux travaux onf confirmé l'hypothèse émise par Fontaine (1967) selon laquelle les gonado tropines et même les hormones thyréotropes de différents Vertébrés constituaient une famille de telles protéines. Du fait de cette homologie et aussi de l'importance évidente, pour l'évolution, de la fonction de reproduction, la biologie comparée des GTH (qui seront surtout considérées ici) et des TSH offre des potentialités exceptionnelles pour le développement des concepts d'une physiologie de l'évolution.

Cette homologie se traduit par des similitudes des structures (isologie) et des effets biologiques (analogie) des GTH des divers Vertébrés. Cependant, une diversification considérable s'est effectuée qui est en particulier responsable, on le verra, de l'existence d'une fréquente spécificité zoologique d'action. Ce phénomène a longtemps retardé les progrès dans la purification puis l'étude des GTH de Vertébrés inférieurs car elle a rendu difficile le dosage biologique de ces dernières. Les méthodes classiques où le receveur est un Mammifère étant en général inutilisables, d'autres approches quantitatives ont dû être inventées sans que la signification physiologique des tests mis en œuvre soit toujours pleinement connue. Diverses techniques de fractionnement des protéines, au long duquel l'activité biologique était ainsi suivie, ont permis d'obtenir des hormones pures. Alors seulement les propriétés physiologiques de ces dernières ont pu être étudiées et de nouvelles méthodes de dosage, plus signifiantes, éventuellement mises au point.

Ces allers et refours nécessaires entre la biochimie et la biologie compliquent aussi l'exposition de nos connaissances actuelles. Nous présenterons d'abord les données concernant la purification ainsi que les propriétés biochimiques et immunologiques des GTH sans détailler les dosages biologiques utilisés qui seront signalés dans la troisième partie consacrée aux effets biologiques des GTH. Auparavant auront été exposés certains résultats concernant la biosynthèse et la sécrétion des GTH ainsi que leur contrôle.

Les données très nombreuses obtenues chez les Mammifères ne pourronł qu'être brièvement résumées ; nous insisterons par contre sur le cas des poissons qui constituent l'objet principal de nos recherches et auxquels leur position phylogénétique confère une importance particulière. Par manque de place certains aspects physiologiques, par exemple les cycles de GTH circulantes, seront laissés de côté malgré leur intérêt (cf. Peter ef Crim, 1979 ; Licht, 1979). Enfin, étant donné l'absence de données précises sur les hormones des Cyclostomes, ce groupe ne sera considéré que dans la discussion.

\section{1. - Nature des hormones}

\section{A. - Purification.}

Des glycoprotéines dovées d'activité gonadotrope ont été purifiées à partir des hypophyses d'une vingtaine d'espèces non mammaliennes représentant les principales classes de Vertébrés (Chondrichthyens ou poissons cartilagineux, Osteichthyens ou poissons osseux, Amphibiens, Reptiles, Oiseaux). Ceci a été mené à bien (références in 
Licht ef al., 1977 ; Fontaine et Burzawa-Gérard, 1978) grâce à l'utilisation de techniques diverses (précipitations fractionnées, chromatographies sur échangeurs d'ions, filtrations sur gels, etc...) dans des conditions très similaires à celles mises en œuvre pour la purification des $\mathrm{LH}$ et $\mathrm{FSH}$ de Mammifères.

En ce qui concerne les Tétrapodes (références in Licht et al., 1977) deux GTH ont généralement été obtenues pour chaque espèce considérée, qui se comportaient respectivement, au cours des purifications, comme une LH ef une FSH mammaliennes. Cependant, une seule hormone a été obtenue à partir d'hypophyses, d'une part de canards ( $a v e c$ à la fois des activités de type FSH et LH) et d'autre part de serpents. Dans ce cas, un facteur capable d'induire l'ovulation chez Xenopus est absent ; la seule GTH mise en évidence pourrait être de type $\mathrm{FSH}$, mais elle ne croise pas dans un RIA de o-FSH (Licht et Bona-Gallo, 1978).

Chez les poissons (références in Fontaine et Burzawa-Gérard, 1978 ; Sumpter et al., 1978a) un certain nombre de résultats ont confirmé l'hypothèse (Burzawa-Gérard, 1974a) qu'il existe chez une espèce donnée une seule GTH exerçant des activités de types LH et FSH. Avant de préciser les caractéristiques biochimiques et immunologiques de ces hormones, signalons que d'autres données suggèrent une situation plus complexe. D'une part en effet, il existerait une différence sexuelle : à partir de la oncGTH, par chromatographie sur diéthylaminoéthyl Biogel, deux fractions ont été séparées qui semblent différer par leurs activités relatives sur l'ovaire et le testicule de truite (Idler et al., 1975b) ; Breton ef al. (1978) ont, quant à eux, obtenu, à partir d'Oncorhynchus (saumons du Pacifique) mâles et femelles respectivement, des GTH douées de certains caractères biochimiques différents, mais de propriétés immunologiques et biologiques similaires. D'autre part, et il s'agit là d'un autre problème, un facteur non glycoprotéique (non adsorbé sur concanavaline A-Sepharose) et donc apparemment d'un type différent de celui des GTH « classiques », a été mis en évidence chez des Téléostéens. Ce facteur, dont la signification physiologique sera discutée plus loin, stimule in vivo l'accumulation de vitellogénine dans les ovocytes, mais est inactif sur la maturation ovocytaire et l'ovulation ; son activité immunologique est 250 fois plus faible que celle de la GTH adsorbée sur concanavaline dans un RIA homologue pour cette dernière ; des formes « légères 》 et « lourdes 》 (poids moléculaires respectivement voisins de 25000 et 45000$)$ ont été mises en évidence ( $\mathrm{Ng}$ eí ldler, 1978a, b). Des données supplémentaires confirmant cette multiplicité des GTH chez les poissons et permetfant son interprétation éventuelle sont clairement nécessaires.

\section{B. - Propriétés biochimiques.}

Un certain nombre de données biochimiques ont été acquises sur les GTH purifiées. Les poids moléculaires sont voisins de 30000 (références in Licht et al., 1977 ; Fontaine et Burzawa-Gérard,1978). En électrophorèse (PAGE), elles donnent en général une zone assez mal délimitée ou plusieurs bandes voisines, un phénomène qui traduit en particulier la microhétérogénéité bien connue de ce type d'hormones. Les compositions globales en acides aminés et en sucres de plusieurs GTH non mammaliennes ont été déterminées (références in Licht et al., 1977 ; Fontaine et Burzawa-Gérard, 1978). Ces compositions (en particulier pour les sucres) varient notablement d'une espèce à l'autre. Licht et al. (1977) ont insisté sur les ressemblances des compositions en acides aminés, d'une part des LH, d'autre part des FSH des divers Tétrapodes ; en fait, l'indice 
d'une différence systématique entre ces deux types d'hormone n'est apparent que pour trois acides aminés (acide glutamique, proline et méthionine) et les GTH de Téléostéens sont de ce point de vue intermédiaires ente LH et FSH. Un trait commun à beaucoup de ces molécules est la richesse en cystine, un paramètre sans doute important pour la structure tertiaire de la molécule et pour son activité ; cependant deux valeurs très faibles ont curieusement été obtenues pour la ti-GTH (Farmer et Papkoff, 1977) et la onc-GTH « $q 》$ (Breton ef al., 1978) (environ 4 versus 8 à 12 par mole chez les autres espèces).

Des progrès importants dans la connaissance des GTH non mammaliennes ont pu être accomplis grâce à la séparation par Burzawa-Gérard (1974b) des deux sousunités de la c-GTH. Après incubation de l'hormone en présence d'urée et chromatographie sur diéthylaminoéthyl Sephadex, deux fractions appelées d'abord SU I et SUII ont été séparées; leurs comportements en Page et en filtration sur Sephadex G100 ont été déterminés ; leurs activités biologiques sont très faibles (ce point a été précisé grâce au test d'accumulation de l'AMPc dans l'ovaire d'anguille in vitro: SU I/c-GTH $<0,001$ et SU II/c-GTH \#0,001. Fontaine-Bertrand, données inédites) ; incubées ensemble, SU 1 et SU II redonnent l'hormone native avec ses caractères de taille moléculaire, de comportement en Page et surtout son activité biologique (Burzawa-Gérard, 1974b).

En collaboration avec $P$. et $J$. Jolles, nous avons pu déterminer la séquence partielle de chacune des sous-unités au $\mathrm{N}$-terminal (Jolles et al., 1977). La séquence partielle de SU I a été comparée à celles des sous-unités de GTH mammaliennes. Lorsqu'on aligne le premier résidu cystine de la c-GTH SU l et ceux des $\alpha$ des Mammifères (łabl. 1) une isologie frappante apparaît, plus de la moitié des résidus étant les mêmes. Une comparaison similaire a été effectuée entre la séquence partielle de la c-GTH SU II ef celles des sous-unités $\beta$ de GTH mammaliennes (tabl. 2). Ici aussi, l'isologie est évidente ; c'est ainsi que 10 résidus sur 26 sont identiques dans c-GTH SU II et h-FSH $\beta$.

\section{TABLEAU 1}

Séquence partielle, au N-ferminal, de la c-GTH SU I ; démonstration de l'homologie entre cefte molécule ef les $\mathrm{LH}_{\alpha}$ ef FSH $\alpha$ de Mammifères (d'après Jolles ef al., 1977 ; pour les hormones de Mammifères, références in Giudice et Pierce, 1978)

c-GTH SU I

h-FSH $\alpha$, h-LH $\alpha$; b-LH $\alpha$ o-LH $\alpha$ su-LH $\alpha$

c-GTH SU I h. $\mathrm{FSH} \alpha$, $\mathrm{h}-\mathrm{LH} \alpha$ b-LH $\alpha$ o-LH $\beta$ su-LH $\alpha$
Tyr-Pro-Arg-Asn-Asp-Met-Asn-Asn-Phe-Gly-Cys-Glu-Glu-Cys-Lys-LeuAla-Pro-Asp-Val-Gin-Asp-Cys-Pro-Glu-Cys-Thr-Leu-

Phe-Pro-Asp-Gly-Glu-Phe-Thr-Met-GIn-Gly-Cys-Pro-GIn-Cys-Lys-LeuThr-Met-GIn-Gly-Cys-Pro-Glu-Cys-Lys-Leu20 30 Lys-Glu-Asn-Asn-Ile-Phe-Ser-Lys-Pro-Gly-Ala-Pro-Val-Tyr-GIn-Cys-MetGIn-Glu-Asp-Pro-Phe-Phe-Ser-GIn-Pro-Gly-Ala-Pro-lle-Leu-GIn-Cys-MetLys-Glu-Asn-Lys-Tyr-Phe-Ser-Lys-Pro-Asx-Ala-Pro-Ile-Tyr-GIn-Cys-MetLys-Glu-Asn-Lys-Tyr-Phe-Ser-Lys-Leu-Gly-Ala-Pro-Ile-Tyr-GIn-Cys-Met- 
L'isologie est plus nette encore relativement aux $\mathrm{LH} \beta$; dans ce cas, les prolines ont en particulier la même position, un résultat sans doute significatif étant donné l'importance de cet acide aminé pour la configuration de la molécule et le fait que les LH connues sont caractérisées par une teneur élevée en proline.

\section{TABLEAU 2}

Séquence partielle, au N-terminal, de la c-GTH SU II ; démonstration de l'homologie entre ceffe molécule ef les $\mathrm{LH} \beta$ ef FSH $\beta$ de Mammifères (d'après Jolles et al., 1977 ; pour les hormones de Mammifères, références in Giudice ef Pierce, 1978)

\begin{tabular}{|c|c|}
\hline & 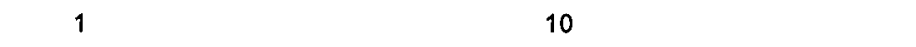 \\
\hline $\begin{array}{l}\text { c-GTH SU II } \\
\text { h-LH } \beta \\
\text { su-LH } \beta\end{array}$ & $\begin{array}{l}\text { Ser- } \theta \text {-Tyr- } \theta \text {-Leu-Pro-Pro- } \theta \text {-Cys-Glu-Pro-Val- X -Glu-Thr-Val- } \\
\text { Ser-Arg-Glu-Pro-Leu-Arg-Pro-Trp-Cys- His-Pro- Ile-Asn-Ala- Ile-Leu- } \\
\text { Ser-Arg-Gly-Pro-Leu-Arg-Pro-Leu-Cys-Glx-Pro- Ile-Asn-Ala-Thr-Leu- }\end{array}$ \\
\hline $\begin{array}{l}\text { O-LH } \beta, \\
\text { b-LH } \beta\end{array}$ & Ser-Ala-Gly-Pro-Leu-Arg-Pro-Leu-Cys-Glx-Pro- Ile -Asn-Ala-Thr-Leu- \\
\hline h-FSH $\beta$ & $\begin{array}{c}\text { Asx-Ser- } \theta-\theta-\theta-\theta-\theta-\theta-\theta-\text { Cys-Glu-Leu-Thr-Asn- Ile -Thr- Ile - } \\
20\end{array}$ \\
\hline $\begin{array}{l}\text { c-GTH SU II } \\
\text { h-LH } \beta \\
\text { su-LH } \beta\end{array}$ & $\begin{array}{l}\text { Ala-Val-Glu-Lys-Glu-Gly-Cys-Pro-Lys-Cys-Leu-Val-Leu- } \\
\text { Ala-Val-Glu-Lys-Glu-Gly-Cys-Pro-Val-Cys- Ile -Thr-Val- } \\
\text { Ala-Ala-Glu-Asp-Glu-Ala-Cys-Pro-Val-Cys- Ile -Thr-Phe- }\end{array}$ \\
\hline $\begin{array}{l}o-\operatorname{LH} \beta \\
b-\mathrm{LH} \beta\end{array}$ & Ala-Ala-Glu-Lys-Glu-Ala-Cys-Pro-Val-Cys- Ile -Thr-Phe- \\
\hline h-FSH $\beta$ & Ala- Ile -Glu-Lys-Glu-Glu-Cys-Arg-Phe-Cys- Ile -Ser- Ile - \\
\hline \multicolumn{2}{|c|}{$0=$ délétion probable. } \\
\hline
\end{tabular}

Ces résultats montrent que c-GTH SU I et SU II sont respectivement les sous-unités $\alpha$ ef $\beta$ de la c-GTH et que cette dernière est plus proche des LH que des FSH de Mammifères.

Dans le cas de l'aci-GTH, une séparation partielle des sous-unités a été menée à bien, après dissociation de l'hormone, grâce à une précipitation fractionnée par le $\mathrm{NaCl}$ à pH 3 (Burzawa-Gérard et Goncharov, 1979).

\section{C. - Hybrides.}

Plusieurs approches ont permis de préciser le degré de similitude entre les GTH (ou leurs sous-unités) provenant de Vertébrés différents : études immunologiques qui seront résumées plus loin et réalisation de molécules hybrides.

Lorsqu'on incube un mélange équimoléculaire de b- $\mathrm{LH} \alpha$ et de c-GTH $\beta$ et étudie en PAGE le comportement du matériel obtenu, on observe l'apparition d'une bande nouvelle, prépondérante, avec un Rf très différent de ceux des sous-unilés utilisées ; de même, en filtration sur Sephadex G100, le pic protéique est élué pour un $K_{D}$ égal à 0,22 bien plus faible que celui des sous-unités. Plus intéressant encore esł le fait que la molécule hybride ainsi mise en évidence manifeste sur la grenoville (test de spermiation) une activité considérable : cette activité est 6 à 8 fois supérieure à celle de chacune des hormones mères, c-GTH ou b-LH ; l'étude de la stimulation de l'adényl cyclase dans un homogénat d'ovaire de grenouille aboutit à une conclusion similaire (Burzawa- 
Gérard et Fontaine, 1976 et données inédites). Ce résultat surprenant suggère que l'hybride pourrait ressembler à une GTH d'Amphibien, c'est-à-dire que les sousunités $\alpha$ et $\beta$ de cette dernière pourraient être respectivement de types mammalien ef pisciaire. La molécule hybride b-LH $\alpha$-c-GTH $\beta$ est très peu active chez un Mammifère; est-elle active chez les poissons ? Une première série d'expériences nous incitait à répondre par la négative car nous n'observions qu'une stimulation très faible de l'adényl cyclase ovarienne in vitro chez le cyprin (Carassius auratus) immature (hybride/c-GTH \#0,014; Burzawa-Gérard et Fontaine, 1976, et données inédites). Dans une seconde série d'expériences, on a utilisé non plus l'incubat des deux sousunités, mais le matériel résultant de la lyophilisation du pic protéique obtenu après filtration de cet incubat sur Sephadex G100 (Burzawa-Gérard, données inédites). Cef hybride purifié (5.277 I) s'est révélé aussi actif que la c-GTH (rapport d'activité $=0,99$, les limites pour $p=0,05$ étant 0,85 ef 1,15 ) sur la concentration de l'AMPc dans l'ovaire d'anguille in vitro (Fontaine-Bertrand et al., données inédites), une expérience qui démontre que l'association b-LH $\alpha$ c-GTH $\beta$ est capable d'exercer une action gonadotrope chez un poisson téléostéen. La contradiction entre ce résultat et celui obtenu lors du dosage de l'AC dans un homogénat d'ovaire de cyprin est évidente et nous y reviendrons. Notons seulement ici qu'elle n'esł pas en rapport avec des différences quantitatives d'activité biologique entre les deux préparations étudiées, l'hybride purifié $(5.277 \mathrm{I})$ manifestant sur la spermiation de la grenouille la même activité (environ $10 \times c-G T H$ ) que l'incubat utilisé d'abord. Une spécificité de reconnaissance, vis-à-vis de la c-GTH, moins marquée chez l'anguille que chez le cyprin ne semble pas non plus pouvoir expliquer les résultats car la c-GTH ef l'hybride sont sensiblement équipotentes sur l'AMPc dans l'ovaire d'un Cyprinidé, la carpe (Fontaine-Bertrand et Salmon, données inédites).

L'électrophorèse analytique et le dosage biologique (spermiation de la grenouille) montrent qu'un hybride similaire à celui dont il vient d'être question se forme entre b-TSH $\alpha$ el c-GTH $\beta$. Par contre aucun indice de formation d'une molécule nouvelle n'a pu jusqu'ici être relevé dans le cas du mélange c-GTH $\alpha$ et b-LH $\beta$.

Des tentatives d'hybridation ont également été effectuées avec des sous-unités de la GTH d'esturgeon. Ici aussi l'électrophorèse analytique suggère que, des deux combinaisons b-LH $\alpha$, aci-GTH $\beta$ et aci-GTH $\alpha$, b-LH $\beta$ seule la première donne un hybride. Par contre, les deux hybrides $\alpha \beta$ possibles se forment bien si on considère les deux poissons, téléostéen et chondrostéen, que sont la carpe ef l'esturgeon (Burzawa-Gérard et Goncharov, 1980). Une telle symétrie dans la possibilité de formation d'hybrides a été démontrée chez les Tétrapodes, entre Mammifères et même entre tortues et Mammifères ; les molécules o- $\mathrm{LH} \alpha-\mathrm{LH} \beta$ de tortue et $0-\mathrm{LH} \beta-\mathrm{LH} \alpha$ de tortue sont, l'une par rapport à l'autre, plus actives respectivement chez la tortue et chez le rat ; cependant la $\mathrm{LH} \alpha$ de tortue n'est pas capable de remplacer parfaitement la o- $\mathrm{LH} \alpha$ tandis que cette dernière confère une activité entière à la LH $\beta$ de tortue (Papkoff et al., 1976 ; Licht et al., 1977, 1978).

L'ensemble de ces données suggère que la sous-unité $\beta$ joue un rôle prépondérant dans le déterminisme de la spécificité zoologique d'action des ensembles $\alpha \beta$, comme elle le fait pour la spécificité fonctionnelle (Pierce ef al., 1971). Cependant, le fait que l'hybride b-LH $\alpha-c-C T H \beta$ soit plus actif sur un Amphibien que la c-GTH met en évidence l'influence de la nature de la sous-unité $\alpha$. 


\section{D. - Propriétés immunologiques.}

1) Antisérums contre des GTH de poissons (ou leurs sous-unités).

Une spécificité élevée des AS dirigés contre des GTH de poissons a été mise en évidence, les hormones de Mammifères (références in Breton et al., 1973) et même de certaines autres espèces de poissons (Tan et Dodd, 1978) étant peu ou non reconnues. Des données plus précises ont récemment été obtenues par des études de liaison et de compétition mettant en jeu des antisérums contre la c-GTH ou chacune de ses sousunités (Burzawa-Gérard et Kerdelhué, 1978 ; Burzawa-Gérard et al., 1980 ; Dufour ef al., 1979). Nous résumerons seulement ici les résultats obtenus avec les AS anti $\mathrm{c}-\mathrm{GTH} \alpha(\mathrm{As} \alpha)$ ou c-GTH $\beta$ (As $\beta$ ) (ceux obtenus avec l'hormone étant intermédiaires). Nous avons déterminé le comportement vis-à-vis de ces antisérums des molécules suivantes : c-GTH et ses sous-unités, het-GTH, aci-GTH, b-LH et ses sous-unités, b-TSH et ses sous-unités, o-FSH et ses sous-unités, ainsi qu'un extrait très partiellement purifié d'hypophyses d'anguilles. En ce qui concerne les études de compétition, seules la c-GTH $\alpha$, la c-GTH et la het-GTH (rappelons qu'il s'agit de l'hormone d'un Cypriniforme comme la carpe) déplacent nettement la ${ }^{125 \mid} \mathrm{c}-\mathrm{GTH} \alpha$ dans le système $\alpha$ ef les régressions obtenues après transformation logit sont alors parallèles. Dans le système $\beta$ (tabl. 3) la situation est bien différente. Toutes les GTH de poisson, ainsi que la b-LH et la b-LH $\beta$ déplacent la ${ }^{125}$ l c-GTH $\beta$, la pente de la régression après transformation logit étant variable. Aucun déplacement n'est observé avec la b-TSH, la o-FSH et leurs sous-unités ; la faible action de la b-LH $\alpha$ est attribuable à une contamination par la b-LH ou la b-LH $\beta$.

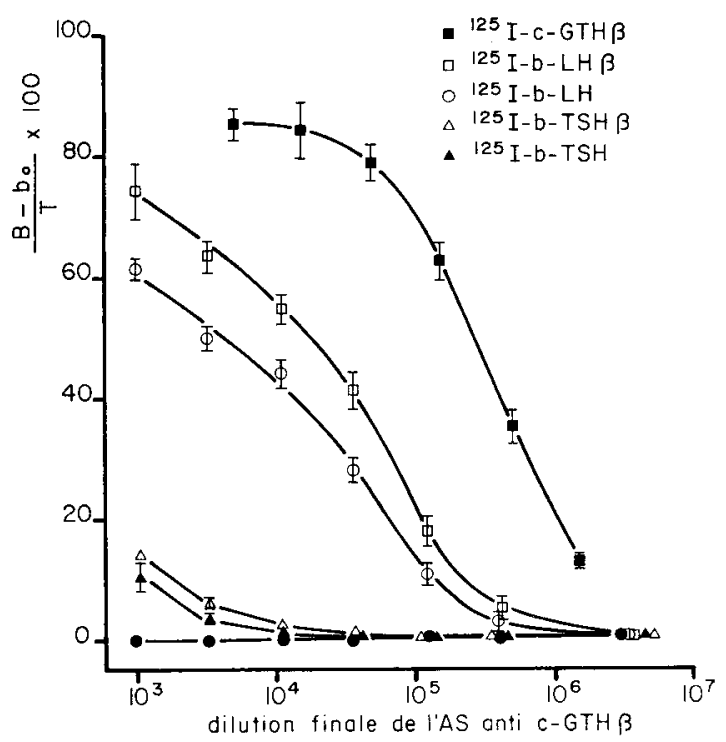

FIG. 1, - Etude de la liaison par un AS anti c-GTHß d'hormones de Mammifères (et de leurs sous-unités) marquées au ${ }^{125}$ / (Dufour ef al., 1979). Les résultats sont exprimés en pourcentage de la radio activité totale $\mathrm{T} ; \mathrm{B}=$ radioactivité liée ; bo = radioactivité liée non spécifiquement (en l'absence d'antisérum).

- : ${ }^{125} \mid$ b-LH $\alpha,{ }^{125} \mid$ b-TSH $\alpha,{ }^{125} \mid$ o-FSH $\alpha,{ }^{125}$ o-FSH, ${ }^{125}$ o-FSH $\beta$. 
Les études de liaison ont été réalisées dans le cas des c-GTH $\alpha$ et $\beta$, des hormones de Mammifères et des sous-unités de celles-ci. Une liaison de la ${ }^{125}$ l c-GTH $\beta$ par l'AS $\alpha$ est mise en évidence et réciproquement. En ce qui concerne les glycoprotéines mammaliennes, l'AS $\alpha$ ne lie nettement que les sous-unités $\alpha$ mammaliennes tandis que l'AS $\beta$ lie, par ordre d'affinité décroissante la $b-\mathrm{LH} \beta$ ef la $b-\mathrm{LH}$, puis la b-TSH $\beta$ et la b-TSH, mais non la o-FSH, la o-FSH $\beta$ ni aucune sous-unité $\alpha$ (fig. 1).

L'AS $\alpha$ manifeste donc une importante spécificité zoologique ; seules les GTH des deux Cyprinidés étudiés entrent en compétition avec la c-GTH $\propto$ mais la réaction croisée est alors complète ; la ressemblance (bien que limitée), démontrée par les études de liaison, entre la c-GTH $\alpha$ et les $\alpha$ mammaliennes, confirme que la première est bien de type $\alpha$.

La spécificité zoologique du système $\beta$ est bien moindre : non seulement toutes les GTH de poissons étudiées mais aussi la b-LH ou la b-LH $\beta$ montrent une réaction croisée. La pente de la régression est d'autant plus faible et différente de celle correspondant à la $c-G T H \beta$ que l'on considère une espèce plus éloignée phylogénétiquement de la carpe. Les études de liaison confirment la parenté de la c-GTH $\beta$ et de la LH $\beta$ et une certaine similifude est même mise en évidence avec la b-TSH $\beta$ mais non avec la o-FSH $\beta$. Ces données sont en accord avec le schéma évolutif proposé par Fontaine et Burzawa-

\section{TABLEAU 3}

Réactions croisées manifestées par diverses hormones ou leurs sous-unités dans un RIA homologue pour la c-GTH $\beta$; comparaison avec leurs activités biologiques chez un poisson (Dufour ef al., 1979)

$\begin{array}{cc}\begin{array}{c}\text { Compétition dans le RIA } \\ \text { pour la c-GTH } \beta\end{array} & \begin{array}{c}\text { Activité }(a) \text { biologique } \\ \text { (Fontaine-Bertrand ef al., } \\ \text { Pente }\left(^{b}\right) \quad \begin{array}{c}\text { Activité }(a) \\ \text { immunologique }\end{array}\end{array}\end{array}$

\begin{tabular}{|c|c|c|c|}
\hline $\begin{array}{l}\text { c-GTH } \ldots \ldots \ldots \cdots \\
\text { het-GTH } \ldots \ldots \cdots \cdots \\
\text { aci-GTH } \ldots \ldots \cdots \cdots \\
\text { b-LH. } \ldots \ldots \cdots \cdots \cdots \\
\text { b-TSH } \ldots \cdots \cdots \cdots\end{array}$ & $\begin{array}{l}-1,20 \\
-0,64 \\
-0,45 \\
-0,34 \\
0\end{array}$ & $\begin{array}{c}1 \\
0,15 \\
0,07 \\
0,005 \\
<10^{-3}\end{array}$ & $\begin{array}{c}1 \\
0,73(0,35-1,52)(c) \\
0,091(0,081-0,105)\left({ }^{c}\right) \\
\quad \leqslant 0,002(e)\end{array}$ \\
\hline ang-GTH $(f) \ldots \ldots$ & $-0,86$ & 0,0014 & $0,0015(0,001-0,002)\left({ }^{c}\right)$ \\
\hline $\begin{array}{l}\text { c-GTH } \alpha \ldots \ldots \ldots \\
\text { b-LH } \alpha \ldots \ldots \ldots \\
\text { b-TSH } \alpha \ldots \ldots \ldots \\
\text { o-FSH } \alpha \ldots \ldots \ldots\end{array}$ & $\begin{array}{c}0 \\
\text { nc } \\
0 \\
0\end{array}$ & $\begin{array}{l}<10^{-3} \\
<10^{-3} \\
<10^{-3} \\
<10^{-3}\end{array}$ & $\begin{array}{c}10^{-3} \\
- \\
-\end{array}$ \\
\hline $\begin{array}{l}\text { c-GTH } \beta \ldots \ldots \ldots \ldots \\
\text { b-LH } \beta \ldots \ldots \ldots \ldots \\
\text { b-TSH } \beta \ldots \ldots \ldots \\
\text { o-FSH } \beta \ldots \ldots \ldots\end{array}$ & $\begin{array}{c}-1,18 \\
-0,38 \\
0 \\
0\end{array}$ & $\begin{array}{c}2,2-2,6\left(^{d}\right) \\
0,005 \\
<10^{-3} \\
<10^{-3}\end{array}$ & $\begin{array}{c}\# 0,0015 \\
- \\
-\end{array}$ \\
\hline
\end{tabular}

(a) L'activité de la c-GTH est prise comme référence. Dans le cas des activités immunologiques, le calcul est effectué à partir des concentrations produisant $50 \mathrm{p} .100$ d'inhibition ; $(b)$ pente des régressions obtenues après transformation logit ; $(c)$ moyenne avec les limites pour $p=0,05 ;\left({ }^{d}\right)$ résultats de deux expériences; $\left({ }^{e}\right)$ l'hormone utilisée dans ce cas est la o-LH; $(f)$ extrait hypophysaire très partiellement purifié.

$\mathrm{nc}=$ non calculable. 
Gérard (1977). La conservation relative, entre poissons et Mammifères, de déterminants antigéniques portés par les $\beta$ nous a suggéré que ces derniers sont au moins pro parte impliqués dans les siructures assurant la liaison des hormones aux récepteurs. Cette hypothèse est renforcée par le fait que les rapports des activités immunologiques de diverses hormones à celle de la c-GTH varient parallèlement avec les rapports des activités biologiques mesurés in vitro sur l'ovaire d'un Téléostéen, l'anguille, par Fontaine-Bertrand ef al. (1978 et données inédites) (tabl. 3).

2) Antisérums contre les GTH de Tétrapodes non mammaliens (ou leurs sous-unités).

La spécificité des antisérums dirigés contre des GTH de grenouille (Daniels et al., 1977), de tortue (Licht ef al., 1977 ; Licht, 1978) et de poulet (Jenkins ef al., 1978) est en général assez stricte, que l'on compare la LH et la FSH de l'espèce considérée ou bien ces dernières et les hormones d'autres classes de Vertébrés. Cependant, une parenté a été mise en évidence entre la LH de tortue d'une part, celles d'Oiseaux et de Mammifères d'autre part (mais non celle de grenouille, ni les GTH de serpents) ; cette parenté est due à une similitude des déterminants antigéniques portés par la sous-unité $\alpha$, une conclusion opposée à celle que nous avons tirée de nos études sur les GTH de poissons ; ce point sera discuté ultérieurement. Par ailleurs, un extrait hypophysaire de poisson cartilagineux produit une réaction croisée intense dans un système aviaire (Scanes et al., 1972).

\section{3) Antisérums contre les GTH mammaliennes.}

Plusieurs données suggèrent que des AS anti o-LH sont capables de reconnaître la c-GTH, mais la réaction, présentant une faible affinité, ne pourrait être mise en évidence que par des méthodes suffisamment sensibles (références in Fontaine, 1975). En immunoélectrophorèse, Pierce ef al. (1976) n'observent effectivement aucune réaction croisée entre des AS anti b-LH $\alpha$ ou b-LH $\beta$ et l'onc-GTH ; lorsque les AS sont préparés contre des antigènes au préalable réduits et S-carboxyméthylés (RCM), l'onc-GTH subissant le même traitement, une réaction nette, bien que non quantifiée, apparaît entre la RMC onc-GTH ef l'AS anti RCM b-LH $\alpha$; même dans ces conditions, aucune réaction croisée n'est mise en évidence entre la b-LH $\beta$ ef l'onc-GTH. Ce dernier résultat peut apparaître en contradiction avec nos données concernant la c-GTH $\beta$ ef la b-LHß (voir supra, Dufour et al., 1979) ; rappelons cependant que la parenté mise en évidence entre ces molécules, par RIA, est quantitativement limitée (rapport d'activité $=0,005$ ) et peut donc ne pas avoir été décelée en immunoélectrophorèse. Quant aux sous-unités $\alpha$ de LH de Mammifères et de poissons, la confrontation des résultats de Pierce et al. (1976) et des nôtres (Burzawa-Gérard et al., 1980) suggère que les déterminants antigéniques communs sont surtout localisés à l'intérieur de ces molécules.

Une absence de réaction croisée a été observée, dans des RIA de LH mammaliennes, avec des GTH de crocodiles, de serpents et d'Oiseaux, mais les GTH de tortues sont, elles, actives. Enfin, les FSH de divers Vertébrés (mais non celles de Squamates *) sont capables de donner une réaction croisée dans des RIA de FSH mammaliennes (références in Licht ef al., 1977 ; Licht et Bona-Gallo, 1978).

\footnotetext{
* Les Squamates groupent les lézards ef les serpents.
} 


\section{II. - Biosynthèse, sécrétion et leur contrôle}

Même chez les Mammifères et a fortiori chez les autres Vertébrés, on sait peu de choses sur les diverses étapes de la synthèse des sous-unités de GTH (formation des chaînes peptidiques, addition des carbohydrates) et sur les mécanismes de la sécrétion des hormones (références in Peter, 1978). Entre ces deux événements intervient l'association des sous-unités et nous insisterons sur une étude comparative récente de la cinétique de ce phénomène (Marchelidon ef al., 1978, 1979, où les références sur cetfe question pourront aussi être trouvées). Il avait été montré dans le cas de LH mammaliennes que la formation de l'hormone à partir des sous-unités était très lente et hautement thermodépendante ; c'est ainsi qu'à $0^{\circ} \mathrm{C}$ aucune association n'était observée pendant $6 \mathrm{~h}$. Chez les poïkilothermes, une telle situation rendrait probablement difficile la synthèse de l'hormone active quand les animaux se trouvent à basse température. La cinétique d'association in vitro des sous-unités de la c-GTH a été étudiée par diverses méthodes (en particulier la spectrophotométrie de différence) qui ont donné des résultałs convergents. Lorsque l'inverse de la concentration en sous-unités libres est portée en fonction du temps, on obtient une droite ; les droites obtenues avec diverses concentrations protéiques initiales sont parallèles : la réaction est de second ordre apparent avec une constante $K$ indépendante de la concentration initiale, comme ceci avait été proposé pour la formation de la o-LH. Le tableau 4 montre que la vitesse d'association des sous-unités est considérablement plus élevée dans le cas de la c-GTH que dans celui de la o-LH ; elle est aussi beaucoup moins thermodépendante. Nous nous sommes demandés quelles étaient les responsabilités respectives de chaque sous-unité dans ces différences et, afin de répondre à cetfe question, nous avons étudié la formation de l'hybride b-LH $\alpha-c-G T H \beta$. La vitesse de formation de cet hybride est intermédiaire entre celle des deux hormones mères, mais le $Q_{10}$ est identique à celui de la formation de la c-GTH (tabl. 4). Ces résultats montrent que des différences de structure de la sousunité $\alpha$ ne modifient pas la thermodépendance de la réaction qui serait donc déterminée par la seule sous-unité $\beta$. Au contraire, les deux sous-unités $(\alpha$ ef $\beta$ ) contribuent à déterminer la vitesse d'association à une température donnée.

\section{TABLEAU 4}

Influence de l'origine zoologique des sous-unifés $\alpha$ ef $\beta$ de GTH sur la cinétique de leur association (données et références concernant lo o-LH, in Marchelidon et al., 1978, 1979)

\begin{tabular}{|c|c|c|c|c|c|}
\hline \multirow[b]{2}{*}{ Température $\left({ }^{\circ} \mathrm{C}\right)$} & \multicolumn{4}{|c|}{$\begin{array}{c}\text { Constante de vitesse } K\left(M^{-1} s^{-1}\right) \text { de la réaction } \\
\text { de second ordre apparent }\end{array}$} & \multirow[t]{2}{*}{$\mathbf{Q}_{10}$} \\
\hline & 4 & 20 & 26 & 37 & \\
\hline$o L H \alpha+o L H \beta \ldots . .$. & $0,07 *$ & 0,86 & 2,1 & 2,4 & $4,8 * *$ \\
\hline c-GTH $\alpha+c-G T H \beta \ldots$ & 78 & 141 & & & 1,5 \\
\hline b-LH $\alpha+c-G T H \beta \ldots$ & 4,8 & 9,8 & & & 1,5 \\
\hline
\end{tabular}

* Valeur obtenue par extrapolation ; ** Déterminé entre 10 et $37^{\circ} \mathrm{C}$. 
Nos connaissances sur la régulation de la biosynthèse ef de la sécrétion des GTH chez les Vertébrés non mammaliens, sont encore limitées. Elles ont été résumées dans une revue de Peter (1978) où les références sur cetfe question pourront être trouvées. Un contrôle hypothalamique de la fonction gonadotrope de l'hypophyse est très vraisemblablement mis en jeu chez les poissons et les divers Tétrapodes. Bien que la LH-RH manifeste une activité chez des représentants de ces groupes, certaines données suggèrent que le facteur hypothalamique mis physiologiquement en jeu pourrait ne pas être le même chez tous les Vertébrés. De plus les résultats de lésions hypothalamiques chez le cyprin ont suggéré la possible existence d'un facteur hypothalamique inhibant la décharge de GTH. Une modulation par les stéroïdes de la sensibilité des cellules gonadotropes aux facteurs de type LH-RH a été mise en évidence chez un poisson et des Oiseaux (Peter, 1978). L'œstradiol stimule chez l'anguille les cellules gonadotropes hypophysaires (Olivereau et Chambolle, 1978). Les dispositions anatomiques hypothalamo-hypophysaires sont très diverses chez les Vertébrés, si bien que les modalités du transfert de l'information de l'hypothalamus à l'hypophyse posent encore de nombreuses questions, en particulier chez les poissons (Sumpter ef al., 1978b; Van Oordt et Ekengren, 1978).

L'importance des facteurs externes (lumière, température, etc...) dans le déterminisme des variations cycliques de la fonction gonadotrope a été bien souvent mise en évidence, par exemple chez des oiseaux (Assenmacher, 1974) ou des poissons (Sundararaj et al., 1978).

\section{III. - Effets biologiques chez les divers Vertébrés}

II est vraisemblable que les GTH des divers Vertébrés exercent au moins une partie de leurs effets par les mécanismes mis en évidence chez les Mammifères et, en tout cas, en se liant d'abord à des récepteurs présents dans les tissus effecteurs. Nous serons amené, en particulier pour tenter de résumer nos connaissances actuelles et aussi dans la discussion, à utiliser ce terme de récepteur (désignant les structures cellulaires qui reconnaissent certaines hormones et dont l'interaction avec ces dernières se traduit par une réponse physiologique des cellules effectrices). Nous sommes conscient que cette utilisation peut se révéler abusive dans certains cas car actuellement, on sait en fait peu de choses sur les récepteurs des GTH chez les Vertébrés inférieurs.

\section{A. - Poissons.}

\section{1. - Poissons osseux (Osteichthyens).}

Par le nombre des espèces, la diversité des milieux habités, ces animaux forment un groupe extrêmement hétérogène où la reproduction, en particulier, peut s'effectuer suivant des schémas très variés. Cette hétérogénéité est évidente, même au sein du Super-Ordre des Téléostéens qui groupe la grande majorité des poissons actuels. Ełant donné le nombre relativement très faible des espèces de Téléostéens éludiées jusqu'ici, les généralisations parfois tentées doivent être considérées, spécialement en ce qui concerne ce groupe, comme des hypothèses de travail. 


\section{a) Effets d'hormones de Téléostéens.}

In vivo, les GTH purifiées de Téléostéens sont en général capables de stimuler les diverses étapes du développement des gonades dont on sait qu'elles sont sous la dépendance de l'hypophyse. La c-GTH et l'onc-GTH simulent chez le mâle la spermatogenèse et la spermiation (par exemple Yamazaki et Donaldson, 1968, Billard et al., 1970 ; Sundararaj et Anand, 1972 ; Nayyar ef al., 1976). La c-GTH augmente l'incorporation de ${ }^{32} \mathrm{P}$ par le testicule immature et l'hydratation de celui-ci (Burzawa-Gérard, 1974a). Chez la femelle, elles stimulent l'ovogenèse, la maturation ovocytaire et l'ovulation (par exemple Yamazaki ef Donaldson, 1968 ; Sundararaj et Anand, 1972 ; Burzawa-Gérard, 1974a ; Sundararaj et al., 1976 ; Fontaine et al., 1976 ; Jalabert et al., 1978 ; Lam ef al., 1978). Ces effets sont au moins en partie médiés par des stéroïdes hormonaux, mais on manque de données précises sur la réponse des taux plasmatiques de ces composés à l'administration de GTH (cf. Fontaine, 1975 ; Billard et al., 1978). Des extraits hypophysaires bruts stimulent la production d'œstradiol $17 \beta$ in vivo (Fostier ef al., 1979) et in vitro (Yaron, communication personnelle). De nombreuses questions restent également posées en ce qui concerne les types cellulaires stéroïdogènes, non seulement dans l'ovaire et le testicule (cf. Lamberi, 1978 ; Callard et al., 1978), mais aussi dans le tissu interrénal, qui pourrait constituer une cible pour la GTH (Sundararaj ef Goswami, 1977).

En ce qui concerne la vitellogenèse, il est généralement admis que la synthèse hépatique de la vitellogénine est stimulée par les œstrogènes (cf. Fontaine, 1975 ; Lam ef al., 1978). Par contre, une controverse intéressante s'est développée au sujet du contrôle hormonal de la fixation de la vitellogénine plasmatique par l'ovocyte. L'apparition de vitellus (et d'une zona radiata) dans les ovocytes, chez des animaux hypophysectomisés, a été mise en évidence sous l'influence de l'onc-GTH ou de la c-GTH chez le cyprin (Yamazaki et Donaldson, 1968 ; Burzawa-Gérard, 1974a), l'anguille (Fontaine et al., 1976) ef le poisson-chat indien (Nath et Sundararaj, 1978). Cependant des études ultrastructurales ont permis à Upadhyay ef al. (1978) de montrer que l'oncGTH, chez la truite (Salmo gairdneri) immature, ne déterminait ni pinocyłose de la vitellogénine plasmatique, ni apparition de zona radiata ou de vésicules vitellines ; ces phénomènes étaient au contraire observés chez des poissons traités par un extrait brut d'hypophyses de saumon du Pacifique (Onchorynchus tshawytscha). Ce résultat suggère qu'un facteur hypophysaire différent de l'onc-GTH purifiée et étudiée jusque-là est nécessaire, dans les conditions expérimentales de ces auteurs, pour l'incorporation de la vitellogénine dans l'ovocyte. Les travaux du groupe d'ldler (cf. $\mathrm{Ng}$ ef Idler, 1978a, b) aboutissent à une conclusion similaire. Avant de considérer ce facteur, pauvre en carbohydrates, comme une GTH, sensu stricto, on doit cependant obtenir les réponses à plusieurs questions. Cette hormone agit-elle directement sur l'ovaire ? Est-elle composée de sous-unités ef homologue des GTH « classiques»? Ces dernières sont-elles réellement inactives sur la vitellogenèse exogène, contrairement aux résultats rappelés plus haut? Le « facteur vitellogénique » n'augmenterait pas la concentration de l'AMPc in vitro dans la gonade de truite ( $\mathrm{Ng}$ et Idler, 1978a) et la très faible stimulation de l'endocytose de vitellogénine observée in vitro par Campbell (1978) demande confirmation. Chez les Amphibiens, où ce problème a été le plus étudié et où l'incorporation de vitellogénine est stimulée par des gonadotropines, la h-CG ne semble exercer 
aucun effet sur cefte incorporation in vitro. Notons aussi que, chez Xenopus, l'environnement hormonal et même, par exemple, le jeûne, peuvent modifier l'effet des gonadotropines (Holland et Dumont, 1975). Chez les poissons un rôle potentialisant des hormones thyroïdiennes (et donc de la TSH) sur la stimulation gonadotrope de la croissance ovarienne et de la vitellogenèse est suggérée par plusieurs travaux (Hurlburt, 1977 ; Lafaurie ef al., 1978).

Le système de l'AMPc est impliqué dans le mécanisme d'action des gonadotropines de Téléostéens purifiées jusqu'ici. La c-GTH et l'onc-GTH stimulent l'activité adényl cyclase dans des homogénats d'ovaire de Cyprins immatures (Fontaine et al., 1970, 1972 ) ; notons que, contrairement à ce qui se passe chez les Mammifères, la stimulation hormonale correspond surtout à une diminution du Km pour l'ATP, plutôt qu'à une augmentation de la Vmax du système enzymatique (Salmon et al., 1974a). Nous n'avons pas connaissance de données détaillées sur les concentrations respectives en ATP - et leurs variations - dans les ovaires de Poissons ef de Mammifères, données qui permettraient de dégager l'éventuelle signification physiologique de cette différence. L'onc-GTH, incubée en présence de fragments de gonade de Truite, y augmente considérablement la concentration en AMPc (Idler ef al., 1975a). La c-GTH exerce la même action sur des fragments d'ovaire d'anguille; à $20^{\circ} \mathrm{C}$ la concentration de $0,01 \mu \mathrm{g} \mathrm{c}-\mathrm{GTH} / \mathrm{ml}$ (qui se situe vraisemblablement dans la gamme des concentrations plasmatiques physiologiques) est déjà active ; la régression réponse-log dose est sensiblement linéaire entre 0,1 et $5 \mu \mathrm{g} \mathrm{c-GTH} / \mathrm{ml}$; enfin la teneur en AMPc est fonction de la durée de l'incubation pendant 20 min au moins : l'absence de temps de latence significatif suggère qu'il s'agit d'une action directe de la c-GTH (Fontaine-Bertrand ef al., 1978). Des modifications des activités enzymatiques du système de l'AMPc sont également induites par un traitement gonadotrope chronique, in vivo (Fontaine et al., 1976). Des variations de la concentration en GMP cyclique ont aussi été mises en évidence dans le testicule de truite sous l'influence d'un traitement gonadotrope (Davis et Smith, 1976). Il apparaît donc probable que, chez les Téléostéens comme chez les Mammifères, les GTH agissent suivant le schéma de Sutherland. Cependant certains résultats paradoxaux ont été obtenus. C'est ainsi que la c-GTH qui provoque, in vivo, le développement sexuel de l'anguille hypophysectomisée et qui est capable, in vitro, de multiplier par 20 la teneur en AMPc de fragments d'ovaire d'anguille, ne stimule pas significativement l'AC dans des homogénats préparés à partir de ce même organe (Fontaine et al., 1976 ; Fontaine-Bertrand ef al., 1978). L'activité protéine kinase (PK) a également été étudiée ; la c-GTH a seulement un effet très faible, in vitro, sur la PK d'ovaire de cyprin. Une différence similaire existe en ce qui concerne l'action de l'AMPc lui-même $\left(10^{-6} M\right)$ sur la PK d'homogénats ovariens ; en présence de théophylline, la PK est multipliée par 5-6 chez le rat mais ne varie pas chez l'anguille ; en absence de théophylline, l'AMPc induit une augmentation significative, mais faible, de la PK de l'ovaire d'anguille (Fontaine et al., 1975 ; Fontaine-Bertrand, données inédites citées dans Fontaine et Burzawa-Gérard, 1978, où ce problème est discuté).

\section{b) Hormones d'autres Vertébrés.}

Quelques données suggèrent que, sur un poisson Téléostéen, les GTH provenant de donneurs appartenant à la même espèce ou au même ordre sont plus actives que 
celles d'autres Téléostéens (références in Fontaine ef al., 1972 ; Breton et al., 1973 ; Fontaine, 1975).

Une spécificité zoologique d'action bien plus nette apparaît dès que l'on considère les GTH de donneurs non Téléostéens. C'est ainsi que l'hormone d'un poisson osseux Chondrostéen, l'esturgeon, est environ 1000 ou 10 fois (une différence discutée ultérieuremenl) moins active que celle de la carpe respectivement sur l'adényl cyclase d'ovaire de cyprin et sur la teneur en AMPc dans l'ovaire d'anguille (Fontaine et al., 1974 ; Fontaine-Bertrand et al., 1978). Au contraire, chez l'esturgeon lui-même (le paramèire étudié étant la maturation ovocytaire in vitro) l'aci-GTH est environ 10 fois plus active que la c-GTH (Goncharov ef al., 1976).

C'est surtout l'effet des hormones de Mammifères qui a été étudié. La b-LH et la o-FSH sont au moins 100 fois moins actives que la c-GTH sur l'adényl cyclase d'ovaire de cyprin (Fontaine et al., 1970). La o-LH augmente significativement, mais faiblement ef à forte dose, la concentration d'AMPc dans l'ovaire d'anguille in vitro (o- $\mathrm{LH} / \mathrm{c}$ GTH $\leqslant 0,002$ ) (Fontaine-Bertrand ef al., 1978). In vivo, les gonadotropines mammaliennes se sont souvent révélées inactives sur la femelle (références in Fontaine, 1975). Chez le poisson-chat indien hypophysectomisé, cependant, si elles ne peuvent déclencher ni l'ovogenèse, ni la formation de vitellus, elles permettent le maintien du poids de l'ovaire et celui des ovocytes déjà chargés de vitellus, la FSH étant moins efficace que la LH (Sundararaj et Anand, 1972 ; Anand et Sundararaj, 1974). LH et FSH stimulent aussi la synthèse de vitellogénine dans le foie, sans doute par l'intermédiaire des œstrogènes (Nath et Sundararaj, 1978). La LH accélère l'ovulation chez le cyprin (Yamazaki, 1965), la maturation ovocytaire ef l'ovulation chez le poisson-chat indien (références in Sundararaj et Goswami, 1977). Enfin, un effet fort intéressant de la o-LH a été mis en évidence chez un poisson hermaphrodite, Monopterus albus (des animaux, non hypophysectomisés ayant cependant été utilisés) : l'hormone stimule intensément, dans l'ovaire, le développement du tissu interstitiel ef l'apparition de lobules testiculaires (Tang et al., 1974).

Chez le mâle, des résultats contradictoires ont également été obtenus. Les gonadotropines mammaliennes se sont révélées impuissantes à restaurer ou à maintenir la spermatogenèse chez plusieurs Téléostéens hypophysectomisés (Billard ef al., 1970 ; Breton ef al., 1973) ; cependant la o-LH exerce ces effets chez le poisson-chat indien hypophysectomisé (références in Nayyar et al., 1976).

Les GTH mammaliennes (surtoul les LH) sont donc capables de produire au moins certains des effets des GTH de Téléostéens mais à des doses bien plus élevées que ces dernières. Une telle spécificité zoologique d'action partielle semble bien en effet de règle aussi bien pour les TSH (Fontaine, 1969) que pour les GTH, on en verra d'autres exemples. Pour expliquer que selon l'espèce, le sexe, l'état physiologique, l'effet final considéré, les hormones mammaliennes apparaissent ou non actives chez les Téléostéens, des hypothèses supplémentaires sont cependant nécessaires : les récepteurs des GTH semblent manifester des exigences quantitatives ou qualitatives qui varient avec ces paramètres. Dans le premier cas, les LH mammaliennes apparaîtraient inactives (sur des paramètres dont la stimulation physiologique requierf une concentration élevée de GTH endogène) simplement parce que les doses utilisées sont trop faibles. II a, par exemple, été signalé chez le poisson-chat indien que la maintenance des ovaires chargés de vitellus nécessitait des niveaux de GTH plus faibles que la stimulation, soit 
de la vitellogenèse, soit de la maturation et de l'ovulation (Sundararaj et Anand, 1972 ; Sundararaj et al., 1976). Cependant de telles différences, lorsqu'elles sont observées in vivo peuvent être en rapport avec des variations non seulement des sensibilités de récepteurs, mais aussi des demi-vies des hormones circulantes.

Dans le second cas, c'est la spécificité même de certaines populations de récepteurs qui se trouverait particulièrement accusée, conduisant à une moindre efficacité des LH mammaliennes sur les effets physiologiques dépendant de la mise en jeu de ces populations de récepteurs. Les résultats d'une expérience effectuée in vitro sur des fragments d'ovaires d'anguilles témoins ou sexuellement développées apportent une indication intéressante sur ce point (Fontaine-Bertrand ef al., données inédites, in Fontaine et Burzawa-Gérard, 1978). Chez l'anguille ayant subi un traitement gonadotrope in vivo, comparée au témoin, la c-GTH est considérablement moins active sur la teneur en AMPc (ceci reflète probablement une désensibilisation de récepteurs consécutive à l'injection in vivo de c-GTH, Salmon, données inédites) ; or la o-LH est alors, au contraire, au moins aussi active. Ceci peut suggérer que la désensibilisation due au traitement par la c-GTH n'a pas affecté certains systèmes récepteurs reconnaissant les LH mammaliennes.

Rappelons enfin que les GTH mammaliennes manifestent une intense activité hétérothyréotrope chez les Téléostéens (Fontaine, 1969).

\section{2. - Poissons cartilagineux (Chondrichthyens).}

Les rares données disponibles proviennent surtout des travaux du groupe de Dodd. Un ensemble de résultats suggère que chez la roussette, l'hormone endogène (scyGTH) stimule la stéroïdogenèse (Sumpter et al., 1978a, b). Craik (1978) a étudié les effets de l'hypophysectomie totale ou partielle sur la disparition de la vitellogénine plasmatique ; ses résultats suggèrent qu'une hormone différente de la scy-GTH (car la seule ablation du lobe ventral, le plus riche en scy-GTH, est sans effet) accélère cetfe disparition. Cependant, aucune modification du taux de synthèse des granules vitellins n'a été observée.

Des hormones gonadotropes de Mammifères (h-CG et PMSG) sont incapables d'atténuer les régressions induites par hypophysectomie dans le testicule de la roussette (Dobson et Dodd, 1977).

Si on fait abstraction des problèmes posés par le contrôle de la vitellogenèse, il apparaît que chez les poissons l'ensemble des récepteurs mis en jeu dans la stimulation hypophysaire des gonades peuvent l'être par une même GTH. Quant à la spécificité zoologique de ces récepteurs, elle est - bien que seulement partielle — toujours très importante. Certains résultats suggèrent qu'elle varie selon l'effet étudié, l'état physiologique du receveur, et qu'il existerait donc chez les poissons, plusieurs classes de récepteurs de GTH.

\section{B. - Amphibiens.}

\section{1. - Effets d'hormones d'Amphibiens.}

Chez le mâle, la FSH et aussi la LH de grenouille stimulent la spermatogenèse. Sur la spermiation, chez Rana catesbeiana, ces deux hormones sont équipotentes. Par 
contre, en ce qui concerne la stimulation de la production d'androgènes par le testicule, la LH apparaît comme l'hormone compétente, chez l'adulte au moins, car elle est alors beaucoup plus efficace aussi bien in vivo qu'in vitro que la FSH (Licht et al., 1977). Cependant, chez des Xenopus immatures, deux groupes de protéines séparés par PAGE ef identifiés respectivement à la $\mathrm{LH}$ et à la FSH de Xenopus augmentent tous deux le taux plasmatique d'androgènes (Stehle-Kahl et Schultheiss, 1980). Chez la femelle, les seules données disponibles concernent la maturation ovocytaire, l'ovulation et la sécrétion de progestérone par l'ovaire. Tous ces phénomènes sont spécifiquement stimulés par la LH et non par la FSH d'Amphibiens (références in Licht ef al., 1977 ; Muller, 1977).

\section{2. - Effets d'hormones d'autres Vertébrés.}

De nombreux travaux ont été effectués à ce sujet el nous en résumerons seulement quelques conclusions principales.

Chez le mâle, aussi bien les GTH de poissons que les LH et FSH de Tétrapodes supérieurs sont toutes actives sur la spermiation (une propriété largement utilisée pour leur dosage biologique) mais elles le sont inégalement et, de plus, leurs activités relatives dépendent du receveur choisi (Burzawa-Gérard et Fontaine, 1972, 1976 ; Licht et al., 1977). Les effets respectifs sur la stéroïdogenèse des FSH et LH de Mammifères comme ceux des hormones d'Amphibiens eux-mêmes, sont encore discutés (Licht ef al., 1977 ; Stehle, 1978 ; Callard ef al., 1978). Licht ef al. (1977) ont obtenu une stimulation de la stéroïdogenèse avec des LH de Mammifères et de Reptiles, mais non avec les FSH correspondantes.

Chez la femelle, les hormones de type LH ou FSH de divers Vertébrés semblent capables, avec des efficacités variables, de stimuler la croissance ovarienne, la sécrétion d'œstrogènes ef la vitellogenèse, y compris l'incorporation de vitellogénine dans l'ovocyte (références in Licht ef al., 1977). Par contre, la maturation ovocytaire et l'ovulation, ainsi qu'une sécrétion accrue de progestérone peuvent être obtenues avec les $\mathrm{LH}$ de divers Tétrapodes (et les GTH de Téléostéens ce qui confirme leur type LH) mais non avec les FSH correspondantes (Goncharov et al., 1976 ; Burzawa-Gérard et al., 1976 ; Licht et al., 1977). Comme sur l'esturgeon lui-même, l'aci-GTH est plus active que la c-GTH sur les Amphibiens, que le test mis en œuvre soit la spermiation in vivo $(2,6 \mathrm{X})$ ou, surtout, la maturation ovocytaire in vitro $(12$ d $28 \mathrm{X})$; en fait, c'est l'efficacité de la c-GTH qui est environ 8 fois moindre sur le second phénomène que sur le premier (Goncharov et al., 1976). L'aci-GTH est aussi légèrement plus active que la o-LH sur la maturation et, contrairement à cette dernière, elle est capable de diminuer l'activité aromatase (Mulner et al., 1978).

Aussi bien chez la femelle que chez le mâle, fout se passe donc comme si plusieurs types de récepteurs des GTH, différant par leur spécificité de reconnaissance, existaient chez les Amphibiens. Les uns apparaissent physiologiquement spécifiques pour la LH: ce sont en particulier ceux mis en jeu chez la femelle pour la stimulation de la sécrétion de progestérone, la maturation ef l'ovulation ; mais ces récepteurs reconnaissent les hormones de type LH des autres Vertébrés. Les autres semblent reconnaître à la fois les LH ef les FSH d'Amphibiens, ainsi que la quasi-totalité des GTH des divers Vertébrés, même si ils le font avec des efficacités variables. 


\section{C. - Reptiles.}

\section{1. - Effets d'hormones de Reptiles.}

L'essentiel des données disponibles provient des travaux du groupe de Licht et nous résumerons seulement ses conclusions (références in Licht ef al., 1977). La maintenance du testicule d'un lézard (Anolis) hypophysectomisé constitue le test utilisé par ces auteurs pour mettre en évidence les FSH de diverses sources. Cependant certaines hormones de type $\mathrm{LH}$ se sont aussi révélées actives sur ce paramètre.

Chez le mâle, aussi bien les FSH que les LH de Reptiles sont capables de stimuler la sécrétion testiculaire d'androgènes. Une certaine spécificité zoologique entre les représentants des ordres étudiés a de plus été mise en évidence

Chez la femelle, l'absence frappante de spécificité vis-à-vis de LH versus FSH est également manifeste. Les FSH et les LH de tortues stimulent in vivo, chez des lézards, la croissance ovarienne, l'ovulation, les structures sexuelles accessoires. Les hormones des deux types augmentent aussi, in vitro, la production de progestérone par le corps jaune ou le follicule préovulatoire de lézard (Crews et Licht, 1975a, b) le site commun d'action étant dans ce cas l'ensemble thèque interne-granulosa. La liaison d'une 125l-FSH de tortue aux gonades de divers Reptiles est inhibée non seulement par des FSH, mais aussi par des LH de tortues (Licht et al., 1977).

Le mécanisme d'action des GTH de Reptiles met probablement en jeu l'adényl cyclase (stimulée par un extrait hypophysaire de tortue dans un homogénat d'ovaire de serpent; Burzawa-Gérard et Fontaine, 1972).

\section{2. - Effets d'hormones d'autres Vertébrés.}

Chez le mâle, diverses GTH de Poissons, d'Amphibiens, d'Oiseaux et de Mammifères se sont en général montrées actives sur le développement du testicule et en particulier sur la sécrétion d'androgènes in vivo et in vifro. Les activités relatives des hormones de types LH et FSH (celles-ci généralement plus efficaces) varient avec leur origine zoologique, avec l'espèce ef l'état physiologique du receveur, avec le mode de traitement (références in Licht et al., 1977 ; Callard et al., 1978). Ces deux types d'hormones exercent-ils leurs effets physiologiques similaires en agissant sur les mêmes cellules et éventuellement grâce à une liaison aux mêmes récepteurs ? Les quelques données disponibles semblent actuellement (cf. cependant Callard et al., 1978) en faveur d'une réponse affirmative au moins partielle. La o-LH et la o-FSH stimulent la production d'androgènes par des cellules de Leydig de lézard (Licht ef al., 1977). La 125l h-FSH se fixe sur les cellules de Leydig et celles des iubes séminifères de divers reptiles et les FSH mammaliennes inhibent cette liaison (Licht et Midgley, 1976). De plus, chez les serpents et les lézards (mais non chez les tortues) les $\mathrm{LH}$ correspondantes ont le même effet. Enfin un ensemble de résultats similaires a été obtenu lorsque le radioligand u'ilisé était une ${ }^{125}$-FSH de tortue (Licht et al., 1977).

Une situation similaire existe chez la femelle, du moins dans le cas des lézards où diverses $\mathrm{LH}$ et $\mathrm{FSH}$, celles-ci généralement plus efficaces, stimulent le développement de l'ovaire, y compris l'ovulation. Une liaison de la ${ }^{125}$ l h-FSH, non seulement aux cellules de la granulosa, mais aussi à d'autres éléments de l'ovaire a été mise en évidence 
(Licht et al., 1977). La o-FSH augmente l'incorporation de thymidine tritiée dans plusieurs catégories cellulaires de l'ovaire du lézard Anolis carolinensis, la o-LH apparaissant inactive (Tokarz, 1978). Lance ef Callard (1978) ont déferminé les variations de stéroìdes plasmatiques in vivo ; chez un serpent, la o-FSH ef la o-LH augmentent l'estradiol, la progestérone et la testostérone, avec des différences selon l'état physiologique du receveur ; chez une tortue, la o-FSH augmente très fortement l'estradiol et la progestérone tandis que la o-LH n'a qu'une action fugace sur l'estradiol. In vitro, la sécrétion de progestérone par l'ovaire d'une tortue est stimulée par la o-FSH mais non par la o-LH, tandis que les deux hormones sont équipotentes sur un crocodile (cf. Licht ef al., 1977 ) ; la biosynthèse des stéroïdes ovariens, in vifro, est modifiée par les LH mammaliennes (Callard ef al., 1976).

Notons enfin que la LH d'Amphibien manifeste une activité hétérothyréotrope chez des Reptiles (Mac Kenzie ef al., 1978).

Chez les Reptiles, les récepteurs des GTH semblent donc, en général, discriminer faiblement les hormones de types FSH et LH, qu'elles proviennent des Reptiles euxmêmes ou d'autres Tétrapodes. II en est en particulier ainsi de ceux mis en jeu chez le mâle pour la production des androgènes et chez la femelle pour l'ovulation. Dans un certain nombre de cas, la FSH semble plus active que la LH correspondante. Des différences importantes ont parfois été notées entre espèces ef notamment entre représentants des Chéloniens, des Squamates et Crocodiliens. C'est ainsi que les récepteurs des GTH semblent, chez les tortues comparées aux lézards, reconnaître moins les LH de Mammifères.

\section{D. - Oiseaux.}

\section{1. - Effets d'hormones d'Oiseaux.}

L'incorporation du ${ }^{32} \mathrm{P}$ dans le testicule du poussin a été le paramètre le plus communément utilisé au cours de la purification des GTH d'Oiseaux ; elle est stimulée aussi bien par les LH que les FSH (Furr et Cunningham, 1970 ; références in Licht ef al., 1977).

Chez le mâle, les effets majeurs des GTH de poulet s'exercent respectivement sur les cellules de Leydig (LH) ou sur la croissance testiculaire et les cellules de Sertoli (FSH) de la caille japonaise (Brown et al., 1975 ; Ishii et Furuya, 1975). La concentration plasmatique de FSH, durant la croissance testiculaire induite par les jours longs, est en étroite corrélation avec le taux de cefte croissance (Follett, 1976). En ce qui concerne la sécrétion des androgènes in vitro, la LH est active, mais des résultats contradictoires ont été obtenus avec la FSH (Licht et al., 1977 ; Maung et Follett, 1977 ; Jenkins ef al., 1978). Etant donné les conditions des expériences, une situation comparable à celle existant chez les Mammifères, où LH et FSH stimulent la stéroïdogenèse dans des types cellulaires différents, pourrait rendre compte de ces contradictions.

Chez la femelle, les résultats de Imai (1973) suggèrent que la $\mathrm{LH}$ et FSH de poulef stimulent le développement folliculaire tandis que la première est bien plus active que la seconde sur l'ovulation. Chez le poulet, à partir de l'âge de 125 jours, la LH de poulet stimule la synthèse d'œstrogènes (Burns, 1972). 


\section{2. - Effets d'hormones d'autres Vertébrés.}

Chez les Oiseaux mâles, les GTH de poissons étudiées se sont révélées actives. L'onc-GTH, la c-GTH et la scy-GTH exercent une action typique sur la sécrétion d'androgènes par des cellules isolées de testicules de cailles. Les rapports d'activité relativement à la o-LH sont respectivement voisins de $0,001,0,02$ et 0,5 . Sur le test plus global de fixation du ${ }^{32} \mathrm{P}$ par le testicule de poulet, ces rapports sont 0,05 (pour la oncGTH) et 0,5 (pour la scy-GTH) (données et références in Jenkins et al., 1978). La oncGTH est incapable d'entrer en compétition avec la ${ }^{225}$ r-FSH pour la liaison à des homogénats de testicules de caille (Ishii et Adachi, 1977).

Des LH d'Amphibiens ou de Reptiles stimulent faiblement (environ 0,005 $\times$ o-LH) la sécrétion d'androgènes par les fragments de testicules de poulet; les FSH correspondantes semblent aussi (Amphibiens) ou moins (Reptiles) efficaces (Licht ef al., 1977). Un extrait d'hypophyses d'Amphibiens inhibe la liaison de la ${ }^{125} \mathrm{r}$-FSH à des testicules de caille (Ishii et Adachi, 1977). Les LH et les FSH de Reptiles déplacent de façon variable $\left(0,01\right.$ à $0,4 \times h$-FSH) la liaison de la ${ }^{125}$ h-FSH à des testicules d'Oiseau ; la FSH est aussi, ou plus, efficace que la LH correspondante (Licht ef al., 1977).

Les GTH de Mammifères (FSH et LH) augmentent la fixation de ${ }^{32} \mathrm{P}$ par le testicule de poulet avec une efficacité voisine de celle des hormones d'Oiseau elles-mêmes. Elles sont capables de stimuler la spermatogenèse et, malgré l'absence de données décisives, on pense en général que les FSH sont, dans ce cas, plus efficaces que les LH (références in Licht et al., 1977). En ce qui concerne la sécrétion d'androgènes par le testicule, un ensemble de données convergentes montrent qu'elle est beaucoup plus sensible aux $\mathrm{LH}$ qu'aux FSH mammaliennes. La o-LH possède même une activité voisine de la $\mathrm{LH}$ de poulet, tandis que les $\mathrm{LH}$ de rat el d'homme sont moins actives ; pour une espèce mammalienne donnée le rapport des activités de la FSH à la LH peut varier de 0,001 à 0,1 (Licht ef al., 1977 ; Maung et Follett, 1977 ; Jenkins et al., 1978). Une certaine spécificité des FSH mammaliennes est également indiquée. Ces hormones, mais non les LH inhibent en effet la liaison de la ${ }^{125}$ l h-FSH ou de la ${ }^{125}$ r-FSH à des testicules d'Oiseaux (Licht et al., 1977 ; Ishii et Adachi, 1977).

Chez les femelles, il semble bien que les LH mammaliennes soient en général moins efficaces que les FSH pour la croissance de l'ovaire, mais le soient plus pour le déclenchement de l'ovulation ; la h-CG, pourtant, serait sans effet sur ce phénomène chez la poule (références in Licht et al., 1977). Shahabi et al. (1975) ont mis en évidence in vivo chez la poule, sous l'influence de la o-LH, une augmentation de la progestérone circulante, aussi bien que de celle présente dans la paroi folliculaire.

Des récepteurs des GTH présents dans les gonades d'Oiseaux présentent donc une certaine spécificité de reconnaissance pour les LH versus les FSH des Oiseaux euxmêmes et sans doute inversement. Comme chez les Mammifères, le premier cas serait par exemple illustré par les récepteurs mis en jeu dans la sécrétion d'androgènes par les cellules de Leydig.

La spécificité zoologique des récepteurs des GTH présents dans les gonades d'Oiseaux (les mâles ayant été les plus étudiés) est faible vis-à-vis des gonadotropines de Mammifères et curieusement, d'un poisson cartilagineux. Elle est relativement 
élevée vis-à-vis des GTH de poissons téléostéens et intermédiaire pour celles d'Amphibiens et de Reptiles. Ces récepteurs semblent en général capables de faire une distinction entre les $\mathrm{LH}$ et les FSH d'autres Vertébrés.

\section{E. - Mammifères.}

\section{1. - Effets des hormones de Mammifères.}

C'est dans cette classe que les données les plus nombreuses ont été rassemblées. Cependant peu d'espèces ont encore été étudiées et, même en ce qui les concerne, la situation n'est pas toujours claire. Il apparaît en particulier que les effets et éventuellement les sifes d'action respectifs des deux gonadotropines $\mathrm{FSH}$ et $\mathrm{LH}$ sont susceptibles de varier avec l'éłape du développement ou l'espèce considérées. En première approximation, on peuf cependant considérer que l'ovulation, la sécrétion de la progestérone par le corps jaune, la sécrétion dans le sang des androgènes par le tissu interstitiel du testicule dépendent principalement de la $\mathrm{LH}$; la FSH est surtout impliquée dans la spermatogenèse et la croissance folliculaire (cf. par exemple Means ef al., 1976 ; Saxena et Rathnam, 1978). La FSH contrôlerait en particulier le nombre de follicules susceptibles d'ovuler (Hirshfield ef Midlgey, 1978).

De nombreuses données indiquent que la plupart au moins de ces effets hormonaux mettent en jeu une liaison de la $\mathrm{LH}$ el de la FSH à des récepteurs membranaires couplés à une adényl cyclase, les récepteurs mis en jeu physiologiquement par LH et FSH étant différents. Quant aux cibles principales de ces hormones, elles semblent bien être chez le mâle les cellules de Leydig (LH) et de Sertoli (FSH, LH), chez la femelle les cellules de la thèque et le corps jaune $(\mathrm{LH})$ et les cellules de la granulosa (FSH puis, en fin de développement du follicule, FSH et $\mathrm{LH}$ ). Cependant des interactions multiples ont été mises en évidence entre $\mathrm{LH}$ et $\mathrm{FSH}$, cette dernière contribuant par exemple dans certains cas à la préparation physiologique de certaines cellules pour une stimulation ultérieure par la LH. De nombreux travaux ont été consacrés ces dernières années au développement ef à la régulation des récepteurs de la FSH et de la $\mathrm{LH}$. Sur ces diverses questions des références peuvent être trouvées, par exemple, dans les articles de Fontaine et al., 1973 ; Thibault, 1977 ; Eshkol et Lunenfeld, 1977 ; Zeleznik et al., 1977 ; Tsafriri et Lindner, 1978 ; Haour et Saez, 1978 ; Lindsey el Channing, 1979). Le degré de spécificité réciproque des récepteurs de chacune des trois hormones glycoprotéiques hypophysaires TSH, FSH, LH est difficile à définir pour plusieurs raisons : interactions physiologiques entre LH ef FSH (par exemple existence possible de récepteurs pour $\mathrm{LH}$ et FSH couplés à une même adényl cyclase), similitudes biochimiques qui rendent difficile l'obtention d'hormones non contaminées, résultats souvent très différents des études de liaison et d'activité biologique. Des sites liant la TSH marquée ont été mis en évidence dans les fractions particulaires de testicule de cobaye (Davies ef al., 1978) ou de rat (Amir ef al., 1978). Chez ce dernier, diverses LH ef préparations de h-CG diminuent cette liaison avec une activité supérieure de deux à quatre ordres de grandeur à leur activité biologique sur la thyroïde. De même, sur ce dernier tissu, diverses GTH et h-CG ont une activité biologique, aussi bien in vivo qu'in vitro, très faible, mais elles inhibent fortement la liaison de la TSH marquée ; pour la h-CG relativement à la b-TSH, l'activité d'inhibition de liaison est de l'ordre de 
1000 fois l'activité biologique (cf. par exemple Amir ef al., 1977 ; Taliadouros et al., 1978). En ce qui concerne les activités biologiques elles-mêmes, la conclusion générale qui se dégage est qu'il existe probablement des réactions croisées, mais qu'elles sont quantitativement faibles (de l'ordre de 10-3).

\section{2. - Effets des hormones d'Osteichthyens.}

On a recherché un effet de la c-GTH dans les systèmes de dosage classiques permettant le dosage spécifique in vivo des LH et FSH mammaliennes, ceux de Parlow (1961) et Steelman et Pohley (1953). Aux doses utilisées, aucun effet n'a été observé : les rapports d'activité c-GTH/o-LH et c-GTH/o-FSH étaient inférieurs à 0,001 (BurzawaGérard, 1974a). Une préparation partiellement purifiée d'onc-GTH s'est également révélée inactive sur la production de progestérone par des cultures de cellules de granulosa d'ovaire de macaque (sensible à la fois aux LH et aux FSH mammaliennes) ; son activité était inférieure à $10^{-4} \times$ o-LH ou o-FSH (Channing ef al., 1974). Cependant, d'autres résultats obtenus ultérieurement par d'autres méthodes donnent une image différente, plus complexe, de la situation. La GTH de Tilapia exerce un effet typique, augmentant avec la dose, sur la production de testostérone par des cellules de

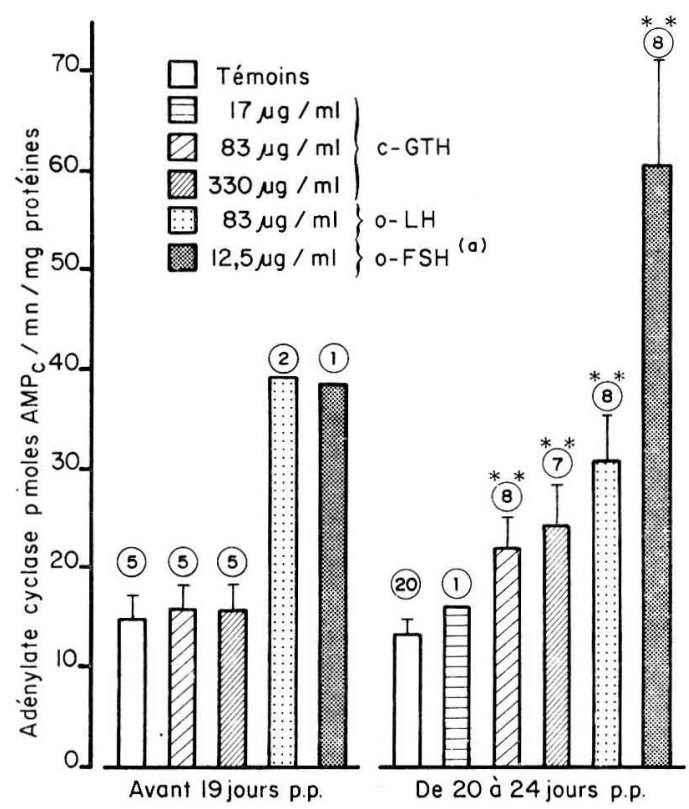

FIG. 2. - Activités comparées de la c-GTH, de la o-LH ef de la o-FSH sur l'adénylate cyclase dans des homogénats d'ovaires de rates âgées de 16 à 18 jours, ou 20 à 24 jours, post-partum (Salmon et al., données inédites).

(a) La préparation de o-FSH utilisée ( $\mathrm{P} 28 a$, environ $7 \times \mathrm{NIH} \mathrm{FSH} \mathrm{SI)} \mathrm{a} \mathrm{été} \mathrm{fournie} \mathrm{par} \mathrm{M.} \mathrm{Jutisz,}$ mais ce sont les concentrations en hormone pure qui sont données ici. Elles ont été calculées en supposant que l'activité de la o-FSH est $45 \times$ NIH FSH SI (cf. Fontaine et al., 1973).

** Stimulation hautement significative $(p<0,01)$.

Le nombre d'expériences est indiqué au-dessus de chaque colonne. La barre correspond à l'erreur standard de la moyenne. (Voir Fontaine et al., 1973, pour les méthodes). 
Leydig de rat, in vitro (un test considéré comme spécifique des $\mathrm{LH}$ ) ; le rapport d'activité ti-GTH/o-LH est voisin de 0,001 (Farmer et Papkoff, 1977). Sur l'adényl cyclase (AC) d'ovaires de rat, la c-GTH (jusqu'à $330 \mathrm{\mu g} / \mathrm{ml}$ ) est inactive chez des rats de moins de 19 jours (c-GTH/o-LH $<3.10^{-4}$; par contre elle manifeste une action faible mais hautement significative dès la concentration de $83 \mu \mathrm{g} / \mathrm{ml}$ chez les animaux de 20 à 24 jours (c-GTH/o-LH \# 7.10-4) (fig. 2) (Salmon ef al., 1980). Or, il s'agit justement de l'âge auquel la stimulation de l'AC par la FSH atteint brusquement un maximum tandis que c'est surtout la LH qui est active auparavant (Fontaine et al., 1973). Il semble donc que la c-GTH soit reconnue par des récepteurs de la FSH et non par ceux de la LH présents chez les rats de 17-18 jours. Cette hypothèse est renforcée par des expériences sur l'inhibition exercée par la c-GTH vis-à-vis des actions de LH et FSH dans le même système (rats de 20-24 jours). En ce qui concerne l'action de la LH, la c-GTH n'inhibe que la stimulation maximale ; les sous-unités c-GTH $\alpha$ et $\beta$ (qui, seules, n'activent pas l'AC) induisent le même type d'inhibition qui apparaît donc comme relativement indépendante d'un effet biologique réel. Par contre, l'action de faibles concentrations de FSH est déjà inhibée par la c-GTH ; dans ce cas l'ensemble des résultats obtenus évoque une interaction de type compétitif (fig. 3) (Salmon et al., 1980). La c-GTH, plus homologue comme on l'a vu, des LH que des FSH mammaliennes, se lierait cependant plus, chez la ratte immature, aux récepteurs des FSH qu'à ceux des LH. Nous suggérons que c'est une spécificité relativement faible des récepteurs des $\mathrm{FSH}$ qui peut rendre compte de ce paradoxe. L'inactivité de la c-GTH sur l'AC d'ovaires de jeunes rats de moins de 19 jours, sensible à la LH, contraste avec les résultats de Farmer et Papkoff (1977) concernant l'action de la ti-GTH sur les cellules de Leydig. Ceci peut

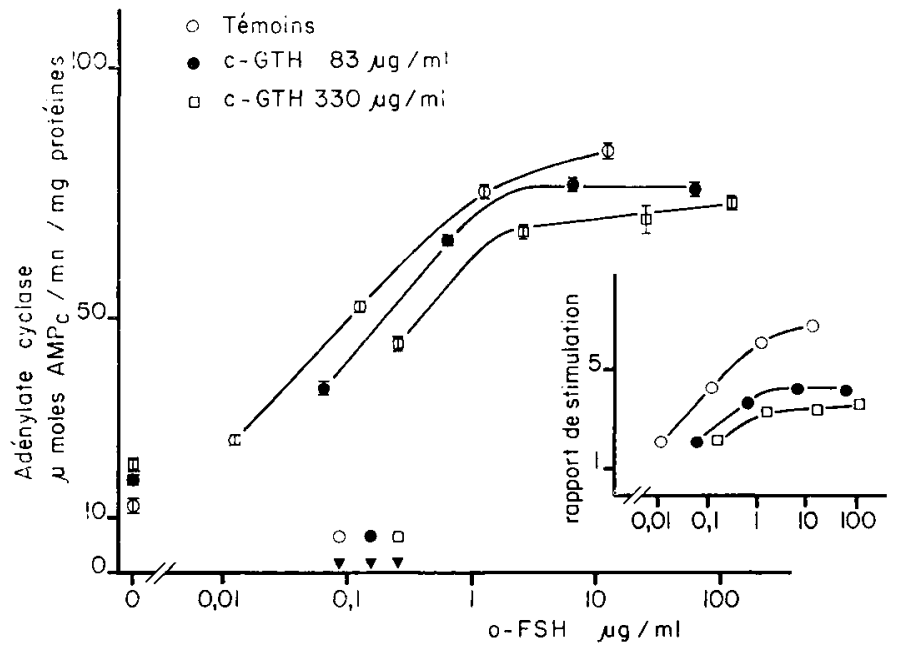

FIG. 3. - Inhibition par la c-GTH de la stimulation par la o-FSH de l'adénylate cyclase dans des homogénats d'ovaire de rates âgées de 21 jours post-partum (Salmon et al., 1980).

Chaque point correspond à la moyenne de triplicats \pm l'erreur standard de la moyenne.

$\checkmark$ : $K_{A}$ apparents pour les trois courbes.

Les concentrations de o-FSH sont celles de l'hormone pure (cf. légende de la fig. 2). Dans l'encart sont donnés les rapports de l'AC stimulée par la FSH à l'AC basale en présence ou non de la c-GTH. (Voir Fontaine ef al., 1973, pour les méthodes.) 
traduire une différence entre les deux hormones de Téléostéens, ou une diminution de la spécificité des récepteurs consécutive, dans le deuxième cas, au traitement des testicules par la collagénase. Les résultats obtenus peuvent aussi refléter une réelle différence de spécificité de reconnaissance des récepteurs de la LH d'une part chez la femelle immature (où elle serait plus stricte) d'autre part chez le mâle adulte.

\section{3. - Effets d'hormones de Tétrapodes non mammaliens.}

Chez la femelle, les activités in vivo de diverses LH non mammaliennes relativement à la o-LH sont faibles et variables ( $<0,003$ pour un Amphibien et des Reptiles ; 0,015 environ pour deux Oiseaux) ; dans le test de Steelman et Pohley (1953), il semble que les FSH de certains Oiseaux et Reptiles aient montré une activilé pouvant atteindre 0,02 à $0,1 \times$ o-FSH (références in Licht ef al., 1977). In vitro, la formation de progestérone par des cellules de la granulosa d'ovaire de macaque est stimulée par une préparation confenant à la fois la LH ef la FSH d'une tortue (environ $0,05 \times$ o-LH ou o-FSH) (Channing et al., 1974). Les cellules de la granulosa d'ovaire de porc lient la 1.2.I h-FSH et cette liaison n'est pas inhibée par des LH mammaliennes ; elle l'est faiblement par les FSH de divers Reptiles ef Oiseaux, mais aussi, curieusement, par certaines LH ; celles d'Alligator et de Chélonia sont aussi, ou même plus actives que les FSH correspondantes (Licht ef al., 1977). Les mêmes auteurs ont étudié la liaison de ${ }^{125 \mid}$ h-LH ou ${ }^{125}$ h-CG à des homogénats d'ovaires de rais pseudoprégnants. Dans ce système, la o-FSH n'interfère que faiblement et il en est de même pour diverses LH non mammaliennes; les FSH correspondantes sont encore environ 10 fois moins efficaces pour l'inhibition de la liaison considérée.

Chez le mâle, deux préparations ont surtout été utilisées, permettant d'estimer in vitro la réponse aux GTH de Tétrapodes inférieurs : coupes de testicules de lapin et cellules de Leydig isolées du testicule de rat (dans les deux cas, les LH mammaliennes stimulent la sécrétion de testostérone, les FSH étant 100 à 2000 fois moins efficaces). Les LH non mammaliennes se révèlent - faiblement mais très significativement actives sur ces tests; elles exercent des actions typiques à des doses environ 100 à 1000 fois plus élevées que les LH mammaliennes. Il est surtout intéressant de noter que le rapport de l'activité de la FSH d'une espèce donnée à celle de la LH de la même espèce est élevé : de 0,03 à près de 1 pour la dinde et deux Amphibiens, alors qu'il est bien plus faible, on vient de le voir, pour les hormones d'un Mammifère (Farmer et al., 1977 ; Licht ef al., 1977).

Chez les Mammifères, la spécificité des récepieurs vis-à-vis de la LH ou de la FSH endogènes apparaît généralement stricte.

L'étude des activités biologiques de GTH de Vertébrés non mammaliens montre que la spécificité zoologique de reconnaissance des récepteurs des GTH est aussi en général très marquée. De plus, elle nous suggère l'hypothèse selon laquelle il existerait plusieurs classes de récepteurs différant par le degré de leur spécificité de reconnaissance. Cette spécificité de reconnaissance serait la plus stricte dans le cas des récepteurs de la $\mathrm{LH}$ chez la femelle, plus imparfaite dans le cas des récepleurs de la $\mathrm{LH}$ chez le mâle et de la FSH chez la femelle. 


\section{Discussion et conclusions}

Les données qui viennent d'être résumées mettent en évidence à la fois l'unité et la diversité des GTH et des mécanismes dans lesquels ces hormones sont impliquées. Cette unité et cette diversité reflètent la phylogenèse ; l'interprétation évolutive des résultats de la biologie comparée, considérée parfois comme du domaine de la philosophie, nous apparaît non seulement comme un outil indispensable, mais aussi comme un but en soi-même. La diversité, enfin, fournit des outils et des modèles ; elle incite à une réflexion originale sur certains concepts.

\section{A. - Aspects évolutifs.}

Refracer l'évolution de caractères biochimiques ou physiologiques constitue une démarche difficile; les réponses aux questions qu'elle pose ne peuvent être encore que partielles et hypothétiques. Cette difficulté est liée à plusieurs raisons, surtout au fait que nous connaissons seulement les résultats actuels de la phylogenèse ; les caractères observés chez les espèces vivantes peuvent être « récents 》 (apomorphes) ou «primitifs » (plésiomorphes), ces derniers présentant un intérêt particulier si on veut retracer les grandes lignes de la macro-évolution. La distinction entre ces deux types de caractères n'est hélas jamais certaine; en ce qui concerne les propriétés biochimiques de protéines homologues et les mécanismes qui les mettent en jeu, nous supposerons que les similitudes ou les identités observées entre des Vertébrés très différents doivent refléter en général des caractères plésiomorphes communs plutôt que des convergences.

La mise en parallèle des données de la biochimie comparée avec celles de la paléontologie, visant à une description de l'évolution biochimique, au sein d'une famille d'hormones par exemple, ne constitue qu'une première étape. Comprendre l'évolution des mécanismes physiologiques qui mettent en jeu ces hormones constitue un second but. Enfin, il serait souhaitable de tenter d'établir des corrélations entre les changements majeurs survenus, chez les Vertébrés, au niveau des diverses fonctions, les grandes inventions comme celles de l'amnios et de l'homéothermie, la conquête de niches écologiques nouvelles. Une telle démarche devrait tendre à préciser les cibles fonctionnelles, sans doute les plus importantes, de la sélection naturelle. II s'agirait alors d'une «physiologie de l'évolution » (au sens que l'on donne à «physiologie du développement $»)$, un vocable provocateur puisque désignant un domaine encore inaccessible à l'expérimentation du nom d'une science expérimentale.

Nous nous limiterons ici à quelques exemples, à quelques hypothèses de travail concernant les GTH, en signalant aussi certaines questions que l'interprétation évolutive conduit à poser. Ces hypothèses sont encore hasardeuses car le nombre d'espèces étudiées est faible et l'hétérogénéité à l'intérieur d'une classe, d'un ordre même, peut être considérable.

1) Les sous-unités $\alpha$ ef $\beta$.

L'ancienneté de la structure sous-unitaire des GTH et TSH en suggère l'importance physiologique. D'un autre côté, l'existence probable à une étape de l'évolution d'une seule molécule ancestrale commune aux deux types de sous-unités conduit à s'interro- 
ger sur la fonction éventuelle de cette molécule, les sous-unités isolées des hormones actuelles semblant inactives. Les différences considérables existant déjà entre la c-GTH $\alpha$ et la c-GTH $\beta$ montrent que les deux lignées $\alpha$ et $\beta$ ont divergé très précocement.

Des parties importantes des séquences des sous-unités $\alpha$ d'une part, $\beta$ de l'autre, sont certainement similaires chez les différents Vertébrés. Il était vraisemblable de supposer que ces structures, les plus conservées, aient joué un rôle, direct ou indirect, pour conférer une activité biologique à la molécule finalement formée, et, d'abord dans l'association des sous-unités $\alpha$ et $\beta$. Il semble bien en être ainsi, mais la phylogenèse a conduit à des changements considérables de la vitesse et de la thermodépendance de l'association $\alpha-\beta$; il serait d'un grand intérêt d'étudier ces paramètres chez divers Vertébrés afin de savoir à quelles étapes de l'évolution a diminué la vitesse et augmenté la thermodépendance. Ces dernières variations observées chez les Mammifères comparés à un poisson, sont-elles liées à l'apparition d'une seconde GTH (de type FSH), au type de contrôle exercé par les facteurs externes et cérébraux, à l'acquisition de l'homéothermie? La lenteur relative de la formation des GTH chez les Mammifères pourrait donner prise à certains mécanismes régulateurs nouveaux ou plus efficaces que chez les Vertébrés inférieurs. La dissymétrie des possibilités d'hybridation entre hormones de poissons osseux et de Mammifères montre aussi que certains sites d'association ont subi des changements importants.

a) Les sous-unifés $\alpha$, au moins chez Mammifères, poissons osseux et sans doute Amphibiens, sont les responsables principales de la spécificité zoologique immunologique : les déterminants antigéniques superficiels n'ont pas eu tendance à être conservés par l'évolution. Ceci suggère que les sous-unités $\alpha$ contribueraient relativement peu à la spécificité de reconnaissance du récepteur. II semble cependant qu'aient été conservées des structures intramoléculaires susceptibles de déterminer une configuration permettant l'association à $\beta$, ce qui témoignerait de l'importance de cette configuration. Par ailleurs, la séquence des 5 acides aminés présents au c-terminal de $\alpha$ est identique chez la carpe et divers Mammifères ef doit donc jouer un rôle fonctionnel.

b) Les sous-unités $\beta$ joueraient un rôle prépondérant dans la détermination des spécificités d'action, fonctionnelle et zoologique et, donc, dans l'association hormonerécepteur. Nous avons précédemment supposé (Fontaine et Burzawa-Gérard, 1977) que les divers types de sous-unités $\beta$ étaient apparues successivement par suite de duplications géniques. Le fait que les types actuels ( $\mathrm{LH}, \mathrm{TSH}, \mathrm{FSH})$ soient tous trois déjà présents chez les Amphibiens est en accord avec l'idée générale que les duplications ont été bien moins nombreuses ultérieurement (Ohno, 1970). Des données variées montrent que les GTH $\beta$ des poissons (l'éventuelle hormone vitellogénique mise à part) font partie de la lignée $L H \beta$. Si on considère la phylogenèse au sein de cette lignée, les études immunologiques chez poissons et Mammifères (Vaitukaitis ef al., 1976) metłent en évidence une parenté des déterminants antigéniques bien plus accusée que dans le cas de la lignée $\alpha$. De plus, les activités immunologique et biologique de diverses hormones vis-à-vis de «systèmes poissons » varient parallèlement. II est vraisemblable que les sifes de liaison aux récepteurs aient été relativement conservés du fait de leur importance physiologique, et ces sites correspondraient, au moins pro parte, aux déterminants antigéniques communs aux LH de poissons et de Mammifères. Une telle 
situation ne semble pas générale, des résultats différents ayant été obtenus chez des Reptiles et des Oiseaux.

Les modalités de l'évolution auraient donc été très différentes pour les $\alpha$ et les $\beta$, témoignant probablement de fonctions différentes des deux types de sous-unités. De plus, pour chacun d'eux, elles auraient varié selon le groupe animal considéré, présentant en particulier des caractères originaux chez les Reptiles.

2) Les hormones gonadotropes ef thyréotropes.

a) Les récepteurs : spécificités fonctionnelle et zoologique.

II semble bien que les diverses GTH et TSH exercent au moins une part de leurs activités par un mécanisme similaire : une liaison à des récepteurs entraînant la stimulation d'une adényl-cyclase. Certains caractères de ce mécanisme ont été conservés des poissons aux Mammifères. Il est frappant par exemple que l'AC ovarienne puisse être stimulée à basse température aussi efficacement chez un Mammifère que chez un poisson (Salmon ef al., 1974b).

L'étude quantitative comparée de la spécificité des interactions entre diverses GTH et TSH et leurs récepteurs, telle qu'on peut l'estimer à partir d'expériences le plus souvent indirectes, est riche d'enseignements. En premier lieu, la spécificité d'un récepteur vis-à-vis de l'hormone physiologiquement compétente n'est jamais totale ; le récepteur reconnaît aussi, mais généralement avec une affinité moindre, d'autres hormones homologues soit présentes chez le donneur où elles jouent un autre rôle (et nous parlerons de la spécificité fonctionnelle du récepteur) soit provenant d'autres Vertébrés (spécificité zoologique). II est frappant de constater que pour de mêmes récepteurs, les deux spécificités, fonctionnelle et zoologique, ne vont pas de pair. Ces différences qui fournissent, on le verra, des renseignements intéressants ne sont pas surprenants; l'existence d'une spécificité zoologique témoigne seulement que la sélection naturelle a permis des changements au niveau des sites de liaison, mais c'esł le degré de spécificité fonctionnelle qui aurait constitué une cible pour cette sélection.

La spécificité zoologique d'action des GTH et TSH implique évidemment des différences entre espèces, non seulement quant à ces molécules, mais aussi quant à leurs récepteurs ; elle implique une évolution de ces deux éléments (cf. par exemple, Fontaine, 1964 ; Licht et al., 1977). La coordination de cette double évolution pose des problèmes très généraux. La question a d'ailleurs souvent été posée de savoir si la duplication génique permettant l'apparition d'une nouvelle protéine fonctionnelle précède ou suit l'apparition de la nouvelle fonction (Zuckerkandl, 1975 ; Orgel, 1977). Dans le cas des GTH et TSH, certains auteurs ont insisté sur l'évolution des hormones, d'autres sur celles des récepteurs. Nous pensons que l'une ou l'autre ont été prépondérantes à des étapes différentes de la phylogenèse. Insistons cependant sur le caractère particulièrement spéculatif des considérations sur l'évolution des récepteurs, puisque nous ne connaissons pas la nature de ceux-ci et ne savons donc rien sur les relations d'homologie qui peuvent ou non exister entre eux, surtout ceux présents dans des tissus différents.

b) Physiologie de l'évolution : un essai. Nous avons suggéré qu'existait, chez des Crâniotes primitifs une seule hormone glycoprotéique hypophysaire qui aurait exercé, à une certaine étape de l'évolution, à la fois des fonctions gonadotrope et thyréotrope 
(Fontaine et Burzawa-Gérard, 1977). Une antériorité de la fonction gonadotrope est supportée par des résultats obtenus chez les lamproies (Pickering, 1972 ; Larsen, 1973), mais la situation pourrait être différente chez les myxines (Kerkof ef al., 1973 ; Henderson et Lorscheider, 1975 ; Matty et al., 1976). Les séquences connues des hormones mammaliennes ont conduit Dayhoff (1976) à proposer l'antériorité d'une TSH. Quoi qu'il en soit, une duplication génique aurait ensuite permis l'apparition d'une seconde lignée $\beta$, et de deux hormones à fonctions distinctes, TSH et GTH, situation que nous supposons exister chez des poissons actuels (Fontaine et Burzawa-Gérard, 1977).

Une stimulation parallèle de la reproduction et de la fonction thyroïdienne qui a été souvent observée, chez les poissons en particulier (références in Fontaine, 1975), pourrait constituer un témoignage des circonstances primitives, mais il est vraisemblable qu'une régulation séparée de la reproduction $(\mathrm{GTH})$ et du développement somatique (TSH) ait constitué un avantage évolutif.

Ce qui est très caractéristique chez les poissons téléostéens actuels, c'est le manque de spécificité zoologique des récepteurs de la TSH, récepteurs qui manifestent pourtanł une spécificité fonctionnelle importante (Fontaine, 1969). Cette situation nous conduit à penser que l'individualisation des fonctions thyréotrope et gonadotrope a été réalisée grâce à une évolution divergente des hormones elles-mêmes, GTH et TSH et à une spécialisation des récepteurs des seules GTH. Certaines hormones de groupes de poissons (Sélaciens, Chondrostéens, Dipneustes) qui n'ont pas rencontré le même succès évolutif que les Téléostéens semblent avoir gardé des caractères plésiomorphes présents encore dans les hormones de Vertébrés supérieurs. Chez les T'éléostéens et en ce qui concerne les GTH, nous avons suggéré qu'il existait chez les poissons plusieurs types de récepteurs, peut-être apparus successivement, pouvant lier la même hormone et que la seconde GTH, de type FSH, apparaissait chez les Amphibiens. Or cette FSH n'exerce apparemment chez ces derniers, aucune fonction qui ne puisse l'être par la LH plus ancienne : la FSH est probablement apparue sans qu'il existe de récepteurs spécifiques pour elle, et sans qu'il existe des récepteurs ne reconnaissant plus l'ancienne hormone. Tout porte à croire que c'est l'évolution des molécules hormonales qui a été le phénomène le plus important. Les récepteurs des G' lH chez les Amphibiens, même ceux qui ne reconnaissent pas la FSH endogène, apparaissent, comme les récepteurs des TSH chez les I'éléostéens, avoir conservé des caractères de non-spécialisation qui rendent compte de leur faible spécificité zoologique. L'avantage évolutif de l'apparition et de la différenciation de la FSH pourrait résider dans le fait que cette hormone n'agit pas sur certains phénomènes comme la maturation ovocytaire ef l'ovulation. Une stimulation indépendante d'autres étapes du cycle sexuel devient donc possible, peut être en rapport avec la conquête encore imparfaite du milieu aérien : la FSH aiderait à résoudre la contradiction entre cette conquête et la nécessité d'une ponte en milieu aquatique.

Chez les Mammifères comparés aux Amphibiens, la spécificité physiologique de récepteurs des FSH et la spécificité zoologique de l'ensemble des récepteurs des GTH sont considérablement accrues. La spécificité zoologique des récepteurs des TSH est beaucoup plus stricte chez les Mammifères que chez les Téléostéens.

Chez les Reptiles, des différences importantes semblent exister entre les trois ordres actuels (qui ont divergé très précocement). En général, la spécificité fonctionnelle des récepteurs des GTH est faible avec peut-être, dans certains cas, perte secon- 
daire d'une de celles-ci, susceptible d'être devenue inutile. Les modalités apparemment particulières de la régulałion hypophysaire des gonades peuvent être en rapport avec l'écophysiologie des Reptiles, particulièrement avec l'importance du contrôle exercé par la température.

En conclusion, il nous semble probable que deux stratégies évolutives aient été mises en jeu.

Dans certains cas (dont peut témoigner la prépondérance actuelle de la spécificité fonctionnelle des récepteurs sur leur spécificité zoologique) l'évolution des protéines hormonales aurait été dominante. II s'agit de l'apparition et de la différenciation, à partir d'une première hormone, de deux lignées distinctes LH et TSH qui prennent chacune en charge certaines des fonctions de l'hormone primitive. Il s'agit aussi de l'apparition de la FSH qui elle-même prend progressivement en charge certaines fonctions de la LH existant auparavant.

Des hormones sont ainsi libérées de certaines tâches, libération qui s'effectuerait par une seconde stratégie, celle de l'évolution des récepteurs. Les récepteurs primitifs des GTH ef TSH auraient été éminemment variés et variables, une situation dont il reste des témoignages, et auraient tendu en général (sauf chez les Reptiles) à une spécialisation progressive. Une spécificité accrue des interactions physiologiques hormone-récepteur apparaît comme une tendance importante de l'évolution.

II est vraisemblable que les deux stratégies distinguées plus haut se soient exercées simultanément dans de nombreux cas, déterminant l'existence de spécificités à la fois zoologique et fonctionnelle importantes. Il semble en avoir été ainsi par exemple pour les GTH au cours de l'épanouissement des Téléostéens et des Mammifères.

\section{B. - Concepts et modèles.}

1) Structure ef fonction des hormones.

L'homologie des GTH et des TSH hypophysaires des différents Vertébrés fait que ces hormones sont autant d'《analogues naturels» au sens pharmacologique du terme. Ceux-ci constituent donc des outils potentiels précieux pour l'étude des relations structure-fonction, en particulier des rôles respectifs des sous-unités $\alpha$ et $\beta$. On a vu que, en l'absence de données structurales complètes, l'étude comparée des propriétés immunologiques et des hybridations pouvait donner des renseignements précieux. Les résultats mettent l'accent sur la différence des fonctions de $\alpha$ et $\beta$ et sur le rôle majeur de cette dernière dans la détermination de la spécificité d'action (fonctionnelle ef zoologique).

2) Variabilité des récepteurs.

Dans l'ovaire d'anguille comme dans plusieurs autres systèmes (cf. par exemple Bérault ef al., 1978) une dissociation est observée entre les effets in vitro de l'hormone compétente, d'une part sur la teneur en AMPc de fragments de l'organe effecteur, d'autre part sur l'activité $A C$ dans des homogénats de cet organe. Plusieurs hypothèses (découplage de l'association récepteur-AC, présence d'inhibiteurs de la liaison de I'hormone au récepteur, homogénéisation de pools d'AC normalement distincts, homogénéisation inadéquate) ont été invoquées pour rendre compte de l'incapacité de l'hormone à stimuler l'AC dans des homogénats. Or, des résultats obtenus avec 
deux analogues de la c-GTH suggèrent que la nature même de l'hormone stimulante est en cause dans cette dissociation. Il s'agit de l'aci-GTH ef de l'hybride b-LH $\alpha-c-G T H \beta$ (qui présentent d'ailleurs d'autres similitudes); les activités de ces deux molécules, relativement à celle de la c-GTH sont 100 à 1000 fois plus élevées, chez des poissons, sur la teneur en AMPc que sur l'activité AC. Tout se passe comme si la spécificité même des récepteurs était différente dans les deux conditions expérimentales.

Cette conclusion rejoint des hypothèses plus générales, suggérées par les éfudes de biologie comparée, concernant la spécificité des récepteurs des GTH et des TSH. Cette spécificité, fonctionnelle et zoologique, n'est que partielle. L'intensité des réactions croisées est variable selon l'hormone et l'effecteur considérés ; elle est, par exemple chez les Mammifères, faible et environ $10^{3}$ fois inférieure à celle des réactions faisant intervenir l'hormone dite compétente ; la « compétence physiologique » est malgré tout, même dans ce cas, seulement relative.

Nous avons été amené de plus à supposer que, chez une espèce donnée, il existe plusieurs catégories de récepteurs capables de permettre l'action de l'hormone compétente, catégories différant par le degré de leur spécificité. Enfin, l'importance relative de ces catégories pourrait varier avec le sexe, l'étape du développement, l'état physiologique ou pathologique. Ces hypothèses sont suggérées par diverses données, qui ont été résumées plus haut, concernant les variations des rapports d'activité entre analogues sur un même effecteur ; rappelons aussi que les thyroïdes de Téléostéens sont stimulables par de nombreuses hormones, en particulier les TSH et GTH de Mammifères (Fontaine, 1969) et que les rapports d'activité (mesurés toutefois in vivo) entre ces hormones et aussi la TSH de Téléostéen sont très variables, en particulier avec la température. Ces récepteurs semblant manifester des spécificités différentes correspondent-ils à des entités différentes ou à des états différents de mêmes entités ? L'ignorance de la structure réelle des récepteurs interdit évidemment toute réponse. L'idée de la variabilité de la spécificité serait compatible avec les conceptions actuelles sur la complexité des systèmes récepteurs ; la spécificité de reconnaissance pourraił dépendre d'un ensemble de structures, protéiques ef phospholipidiques, de l'environnement membranaire qui lui-même est sans doute variable.

De tels phénomènes pourraient rendre compte en partie de cerłains résultats expérimentaux, par exemple, la discordance entre les effets d'hormones dans des homogénats et sur des fragments d'organes, celle entre les activités hormonales mesurées par activation de l'AC ou par inhibition de liaison (par exemple Amir et al., 1977), la diminution de la spécificité de réaction, vis-à-vis de la h-LH, du corps jaune humain après congélation (Rice, 1978) et éventuellement d'observations effectuées en pathologie.

3) Des espèces pour des problèmes.

Certaines espèces présentent des cycles biologiques, et en particulier reproducteurs, exceptionnels, et sont susceptibles de fournir des modèles pour l'étude de certains aspects de la physiologie de la reproduction. Les Oiseaux ont représenté et représentent un matériel de choix ; rappelons seulement la rapidité du développement testiculaire sous l'influence de la lumière ; un des mécanismes impliqués, même s'il est secondaire, pourrait être une auto-induction des récepteurs de la FSH (Tsutsui ef Ishii, 
1978) ; la démonstration de ce phénomène est facilitée par l'utilisation d'une espèce où il est exacerbé. L'anguille européenne ne se reproduit qu'après sa migration en mer des Sargasses, qui suit un séjour de 10 à 20 ans dans les eaux douces continentales. Pendant tout ce séjour l'anguille jaune, puis argentée, reste immature sexuellement, les raisons de ce bloquage (sans doute central, mais peut-être aussi ovarien chez l'anguille jaune) étant encore inconnues. Il est probable que cette espèce pourrait constituer un modèle intéressant pour l'étude du déterminisme de la puberfé. Même chez les Mammifères enfin, on sait combien les cycles reproducteurs sont variés et combien l'étude de diverses espèces sauvages ou domestiques a permis de progrès.

Chez les poissons Téléostéens, une seule GTH exercerait les diverses activités gonadotropes (peut-être à l'exception de la fixation de vitellogénine par l'ovocyte). Cette situation très particulière a suggéré que certains problèmes comme la physiologie des récepteurs gonadiques pouvaient y être avantageusement étudiés ; il est vraisemblable que le contrôle par l'hypothalamus, le cerveau et les facteurs du milieu extérieur (contrôle sur lequel des données intéressantes ont déjà été obtenues) y revêt aussi des caractères originaux. En ce qui concerne les facteurs de décharge hypothalamiques en particulier, l'étude des Vertébrés inférieurs a apporté et apportera certainement des résultats originaux. Elle a conduit à les considérer comme des neuro-transmetteurs, mais ils pourraient aussi avoir d'autres propriétés. Rappelons que chez un Amphibien, groupe où le TRF ne semble pas stimuler la décharge de TSH, une concentration considérable du « neuropeptide » a été mise en évidence dans la peau (Jackson et Reichlin, 1974).

Il est frappant, enfin, de constater que les deux phénomènes biologiques principaux que sont le développement somatique et la reproduction sont régulés, même si ce n'est que pro parte et indirectement, par des hormones homologues GTH et TSH (une des actions majeures des hormones thyroïdiennes chez les Vertébrés inférieurs s'exerçant sur la croissance et certaines métamorphoses). Une coordination entre le développement et la reproduction esł réalisée suivant des modèles éminemment variables selon les Vertébrés. Une étude comparée de cette coordination et de ses mécanismes serait susceptible d'être fructueuse pour la biologie de la reproduction et du développement.

Présenté au Colloque D.G.R.S.T. de Porf Boil, 27 février-1 er mars 1979.

Accepté en septembre 1979.

Remerciements. - Nombre des résultałs et des idées résumés dans cette revue reflètent le travail d'une équipe qui comprend, au sein du Laboratoire de Physiologie générale et comparée du Muséum, Mmes E. Burzawa-Gérard, N. Delerue-Le Belle, S. Dufour, A. Dumas, E. Fontaine-Bertrand, MM. J. Marchelidon et C. Salmon. Ce travail n'aurait pu être mené à bien sans l'aide du Comité de l'Action concertée de la DGRST « Biologie de la Reproduction ef du Développement» (contrats no 72-70044, 74-70515 ef 77-70649). En plus des collègues d'autres laboratoires dont la collaboration a permis des publications communes, Mme F. Xavier, MM. R. Mornex, R. Ozon ef J. Roffi ont bien voulu aussi participer à des discussions enrichissantes. Des hormones gonadotropes et thyréotropes purifiées de Mammifères, ainsi que leurs sous-unités, ont été 
fournies par MM. M. Jutisz (CNRS, Gif-sur-Yvette, France), J. Pierce (UCLA, Los Angeles, USA), M. Sairam (Institut de Recherches cliniques, Montréal, Canada). Des préparations hormonales standards ont été fournies par « The Endocrine Study Section », NIH, USA. Mme N. Delerue-Le Belle a apporté son aide à l'établissement de la liste de références et à la correction du manuscrit qui a été dactylographié par Mme F. Lieron.

\section{Références}

AMIR S. M., UCHIMURA H., INGBAR S. H., 1977. Interactions of bovine thyrotropin and preparations of human chorionic gonadotropin with bovine thyroid membranes. J. clin. Endocrinol. Metab., 45, 280-292.

AMIR S. M., SULLIVAN R. C., INGBAR S. H., 1978. Binding of bovine thyrotropin to receptors in Rat testis and its interaction with gonadotropin. Endocrinology, 103, 101-111.

ANAND T. C., SUNDARARAJ B. I., 1974. Ovarian maintenance in the hypophysectomized catfish, Heteropneustes fossilis (Bloch) with mammalian hypophyseal and placental hormones and gonadal and adrenocortical steroids. Gen. comp. Endocrinol., 22, 154-168.

ASSENMACHER I., 1974. External and internal components of the mechanism controlling reproductive cycles in Drakes, 197-248. In PENGELLEY E. T., Circannual clocks, Annual biological rhythms, Acad. Press.

BERAULT A., THEOLEYRE M., JUTISZ M., 1978. Is C-AMP and intermediate in the releasing action of Gn-RH? Ann. Endocrinol., 39, 227-228.

BILLARD R., BURZAWA-GÉRARD E., BRETON B., 1970. Régénération de la spermatogenèse du cyprin hypophysectomisé (Carassius auratus L.) par un facteur gonadotrope hautement purifié de carpe. C. R. Acad. Sci. Paris, sér. D, 271, 1896-1899.

BILLARD R., BRETON B., FOSTIER A., JALABERT B., WEIL C., 1978. Endocrine control of the teleost reproductive cycle and its relation to external factors : salmonid and cyprinid models, 37-48. In GAILLARD P. J., BOER H. H., Comparative Endocrinology. Elsevier/North Holland Biomed., Press, Amsterdam.

BRETON B., BILLARD R., JALABERT B., 1973. Specificité d'action el relations immunologiques des hormones gonadotropes de quelques Téléostéens. Ann. Biol. anim. Bioch. Biophys., 13, 347 362.

BRETON B., PRUNET P., REINAUD P., 1978. Sexual differences in salmon gonadotropin. Ann. Biol. anim. Bioch. Biophys., 18, 759-765.

BROWN N. L., BAYLE J. D., SCANES C. G., FOLLETT B. K., 1975. Chicken gonadotrophins : their effects on the testes of immature and hypophysectomized Japanese quail. Cell Tiss. Res., 156, 499-520.

BURNS J. M., 1972. The influence of chicken and mammalian luteinizing hormones on ovarian estrogen synthesis in the pullet (Gallus domesticus). Comp. Biochem. Physiol., 42A, 1011-1017.

BURZAWA-GÉRARD E., 1974a. Etude biologique et biochimique de l'hormone gonadotrope d'un poisson Téléostéen, la carpe (Cyprinus carpio L.). Mém. Mus. nat. Hist. nat., sér. A, 86, 1-77.

BURZAWA-GÉRARD E., 1974b. Séparation et réassociation des sous-unités de l'hormone gonadotrope d'un poisson Téléostéen, la carpe (Cyprinus carpio L.). C.R. Acad. Sci. Paris, sér. D., 279, 1681-1684.

BURZAWA-GÉRARD E., FONTAINE Y. A., 1972. The gonadotropins of lower Vertebrates. Gen. comp. Endocrinol., suppl. 3, 715-728.

BURZAWA-GÉRARD E., FONTAINE Y. A., 1976. Formation d'une molécule hybride douée d'une activité gonadotrope sur la grenouille à partir de la sous-unité $\alpha$ de l'hormone lutéinisante bovine et d'une sous-unité de l'hormone gonadotrope d'un poisson Téléostéen. C. R. Acad. Sci. Paris, sér. D, 282, 97-100.

BURZAWA-GÉRARD E., GONCHAROV B. F., 1980 . Les sous-unités de l'hormone gonadotrope d'un Chondrostéen, l'esturgeon (Acipenser stellatus) : séparation partielle et molécules hybrides. Gen. comp. Endocrinol. (sous presse). 
BURZAWA-GÉRARD E., KERDELHUÉ B., 1978. Ełude par radioimmunologie des propriétés des immunsérums de l'hormone gonadotrope de la carpe (Cyprinus carpio L.) et de ses sousunités. Ann. Biol, anim. Bioch. Biophys., 18, 773-780.

BURZAWA-GÉRARD E., GONCHAROV B. F., DUMAS A., FONTAINE Y. A., 1976. Further biochemical studies on carp gonadotropin (c-GTH). Biochemical and biological comparison of c-GTH and a gonadotropin from Acipenser stellatus Pall. Gen. comp. Endocrinol., 29, 498505.

BURZAWA-GÉRARD E., DUFOUR S., FONTAINE Y. A., 1980. Relations immunologiques entre les hormones glycoprotéiques hypophysaires de quelques poissons ef de Mammifères. Gen. comp. Endocrinol (sous presse).

CALLARD I. P., MCCHESNEY I., SCANES C., CALLARD G. V., 1976. The influence of mammalian and avian gonadotropins on in vitro ovarian steroid synthesis in the turtle (Chrysemis picta). Gen. comp. Endocrinol., 28, 2-9.

CALLARD I. P., CALLARD G. V., LANCE V., BOLAFFI J. L., ROSSET J. S., 1978. Testicular regulation in non-mammalian Vertebrates. Biol. Reprod., 18, 16-43.

CAMPBELL C. M., 1978. In vifro stimulation of vitellogenin incorporation into trout oocytes by salmon pituitary extracts. Ann. Biol. anim. Bioch. Biophys., 18, 1013-1018.

CHANNING C. P., LICHT P., PAPKOFF H., DONALDSON E. M., 1974. Comparative activities of mammalian, reptilian and piscine gonadotropins in monkey granulosa cells. Gen. comp. Endocrinol., 22, 137-145.

CRAIK J. C. A., 1978. Effects of hypophysectomy on vitellogenesis in the Elasmobranch Scyliorhinus canicula L. Gen. comp. Endocrinol., 36, 63-67.

CREWS D. P., LICHT P., 1975a. Stimulation of in vitro steroid production in turtle ovarian tissue by reptilian, amphibian and mammalian gonadotropins. Gen. comp. Endocrinol., 27, 71-83.

CREWS D. P., LICHT P., 1975b. Site of progesterone production in the reptilian ovarian follicle. Gen. comp. Endocrinol., 27, 553-556.

DANIELS E. L., LICHT P., FARMER S. W., PAPKOFF H., 1977. Immunochemical studies on the pituitary gonadotropins (FSH and LH) from the bullfrog Rana Catesbeiana. Gen. comp. Endocrinol., 32, 146-157.

DAVIES T. F., SMITH B. R., HALL R., 1978. Binding of thyroid stimulators to guinea pig testis and thyroid. Endocrinology, 103, 6-10.

DAVIS J., SMITH M., 1976. Changes in trout testis cyclic GMP concentration during hormonally induced spermatogenesis. J. cyclic Nucleotide Res., 2, 365-372.

DAYHOFF M. O., 1976. The origin and evolution of protein superfamilies. Fed. Proc., 35, 2132-2138.

DOBSON S., DODD J. M., 1977. Endocrine control of the testis in the dogfish Scyliorhinus canicula L. I. - Effects of partial hypophysectomy on gravimetric, hormonal and biochemical aspects of testis function. Gen. comp. Endocrinol., 32, 41-52.

DUFOUR S., BURZAWA-GÉRARD E., FONTAINE Y. A., 1979. Evolution des hormones glycoprotéiques hypophysaires : données radioimmunologiques sur les sous-unités de l'hormone gonadotrope de la carpe (Cyprinus carpio L.). C. R. Acad. Sci., Paris, sér. D., 289, 137-140.

ESHKOL A., LUNENFELD B., 1977. Control of follicular development : induction of FSH and LH receptors, 318-323. In JAMES W. H. T., Endocrinology, vol. 1, Excerpła med., Amsterdam.

FARMER S. W., PAPKOFF H., 1977. A teleost (Tilapia mossambica) gonadotropin that resembles luteinizing hormone. Life Sci., 20, 1227-1232.

FARMER S. W., SUYAMA A., PAPKOFF H., 1977. Effect of diverse mammalian and non-mammalian gonadotropins on isolated rat Leydig cells. Gen. comp. Endocrinol., 32, 488-494.

FLORKIN M., 1966. Aspects moléculaires de l'adaptation et de la phylogénie. Masson, Paris.

FOLLETT B. K., 1976. Plasma follicle-stimulating hormone during photoperiodically induced sexual maturation in male Japanese quail. J. Endocrinol., 69, 117-126.

FONTAINE Y. A., 1964. Characteristics of the zoological specificity of some protein hormones from the anterior pituitary. Nature, 202, 1296-1298.

FONTAINE Y. A., 1967. Spécificités des glycoprotéines hypophysaires à activité thyréotrope et hypothèses sur leur phylogénie. Bull. Soc. Chim. biol., 49, 647-655.

FONTAINE Y. A., 1969. La spécificité zoologique des protéines hypophysaires capables de stimuler la thyroïde. Acta endoc., 60, suppl. 136, 1-154. 
FONTAINE Y. A., 1975. Hormones in Fishes, 139-212. In MALINS D. C., SARGENT J. R., Biochemical and biophysical perspectives in marine biology, Acad. Press N. Y.

FONTAINE Y. A., BURZAWA-GÉRARD E., 1977. Esquisse de l'évolution des hormones gonadotropes et thyréotropes des Vertébrés. Gen. comp. Endocrinol., 32, 341-347.

FONTAINE Y. A., BURZAWA-GÉRARD E., 1978. Biochemical and biological properties of fish gonadotropins and their subunits : comparison with mammalian hormones, 361-380. In MC KERNS K. W., Structure and function of the gonodotropirs, Plenum Press, N. Y.

FONTAINE Y. A., BURZAWA-GÉRARD E., DELERUE-LE BELLE N., 1970. Stimulation hormonale de l'activité adényl cyclasique de l'ovaire d'un poisson téléostéen, le cyprin (Carassius auratus). C. R. Acad. Sci., Paris, sér. D, 271, 780-783.

FONTAINE Y. A., SALMON C., FONTAINE-BERTRAND E., BURZAWA-GÉRARD E., DONALDSON E. M., 1972. Comparison of the activities of two purified fish gonadotropins on adenyl cyclase activity in the goldfish ovary. Can. J. Zool., 50, 1673-1676.

FONTAINE Y. A., SALMON C., FONTAINE-BERTRAND E., DELERUE-LE BELLE N., 1973. Agerelated changes in $\mathrm{FSH}$ - and in $\mathrm{LH}$ - sensitive ovarian adenyl cyclase from prepubertal rats. Horm Metab. Res., 5, 376-380.

FONTAINE Y. A., BURZAWA-GÉRARD E., SALMON C., FONTAINE-BERTRAND E., DELERUELE BELLE N., 1974. Pituitary gonadotropins and their stimulation of ovarian adenyl cyclase activity : comparative studies in fish and mammals, 351-360. In RASTOGI G. K., Proc. 5th Asia Oceania Congr. Endacrincl., Endocrine Soc. of India, vol. 1, Chandigarh.

FONTAINE Y. A., FONTAINE-BERTRAND E., DELERUE-LE BELLE N., 1975. Croissance de l'ovaire et enzymes du système de l'AMP cyclique. J. Physiol. Paris, 71, 134A.

FONTAINE Y. A., LOPEZ E., DELERUE-LE BELLE N., FONTAINE-BERTRAND E., LALLIER F., SALMON C., 1976. Stimulation gonadotrope de l'ovaire chez l'anguille (Anguilla anguilla). hypophysectomisée : morphologie, activités adényl cyclase et phosphodiestérase de I'AMP cyclique. J. Physiol. Paris, 72, 871-892.

FONTAINE-BERTRAND E., SALMON C., FONTAINE Y. A., 1978. Effet d'hormones gonadotropes, in vifro, sur la concentration de l'adénosine monophosphate cyclique dans l'ovaire de l'anguille (Anguilla anguilla L.). Ann. Biol. anim. Bioch. Biophys., 18, 805-811.

FOSTIER A., BRETON B., JALABERT B., 1979. Hypophysial stimulation of œstradiol $17 \beta$ secretion in carp, Cyprinus carpio L. Ann. Endocrinol., 40, 83-84.

FURR B. J. A., CUNNINGHAM F. J., 1970. The biological assay of chicken pituitary gonadotrophins. Br. Poult. Sci., 11, 7-13.

GIUDICE L. C., PIERCE J. G., 1978. Glycoprotein hormones : some aspects of studies of secondary and tertiary structure, 81-110. In Mc KERNS K. W., Structure and function of the gonadotropins, Plenum Press, N. Y.

GONCHAROV B., BURZAWA-GÉRARD E., FONTAINE Y. A., 1976. L'étude comparative de l'effeł biologique des gonadotropines purifiées de la carpe (Cyprinus carpio) et de l'esturgeon (Acipenser stellofus). Ontogenesis, 7, 85-89 (en russe).

HAOUR F., SAEZ J. M., 1978. Leydig-cell responsiveness to LH-hCG stimulation : mechanisms of hCG- and steroid-induced refractoriness, 497-516. In Mc KERNS K. W., Structure and function of the gonadotropins, Plenum Press, N. Y. and London.

HENDERSON N. E., LORSCHEIDER F. L., 1975. Thyroxine and proteinbound iodine concentrations in plasma of the pacific hagfish, Eptatretus stouti (Cyclostomata). Comp. Biochem. Physiol., 51 A, 723-726.

HIRSHFIELD A. N., MIDGLEY A. R., Jr, 1978. The role of FSH in the selection of large ovarian follicles in the rat. Biol. Reprod., 19, 606-611.

HOLLAND C. A., DUMONT J. N., 1975. Oogenesis in Xenopus laevis (Dandin). IV. - Effects of gonadotropin, estrogen, and starvation on endocytosis in developping oocytes. Cell Tiss. Res., $162,177-184$.

HURLBURT M. E., 1977. Role of the thyroid gland in ovarian maturation of the goldfish, Carassius auratus L. Can. J. Zool., 55, 1906-1913.

IDLER D. R., HWANG S. J., BAZAR L. S., 1975a. Fish gonadotropin(s). I. - Bioassay of salmon gonadotropin(s) in vitro with immature trout gonads. Endocr. Res. Comm., 2, $199-$ 213. 
IDLER D. R., BAZAR L. S., HWANG S. J., 1975b. Fish gonadotropin(s). III. - Evidence for more than one gonadotropin in Chum salmon pituitary glands. Endocr. Res. Comm., 2, 237-249.

IMAI K., 1973. Effects of avian and mammalian pituitary preparations on induction of ovulation in the domestic fowl, Gallus domesticus. J. Reprod. Fertil., 33, 91-98.

ISHII S., ADACHI T., 1977. Binding of avian testicular homogenate with rat follicle stimulating hormone and inhibition of the binding by hypophyseal extracts of lower Vertebrates. Gen. comp. Endocrinol., 31, 287-294.

ISHII S., FURUYA T., 1975. Effects of purified chicken gonadotropins on the chick testis. Gen. comp. Endocrinol., 25, 1-8.

JACKSON I. M. D., REICHLIN S., 1977. Thyrotropin-releasing hormone : abundance in the skin of the frog, Rana pipiens. Science, 198, 414-415.

JALABERT B., BRETON B., FOSTIER A., 1978. Precocious induction of oocyle maturation and ovulation in rainbow trout (Salmogairdnerii). Problems when using $17 \alpha$-hydroxy $20 \beta$ dihydroprogesterone. Ann. Biol. anim. Bioch. Biophys., 18, 977-984.

JENKINS N., SUMPTER J. P., FOLLETT B. K., 1978. The effects of Vertebrate gonadotrophins on androgen release in vitro from testicular cells of japanese quail and a comparison with their radioimmunoassay activities. Gen. comp. Endocrinol., 35, 309-321.

JOLLES J., BURZAWA-GÉRARD E., FONTAINE Y. A., JOLLES P., 1977. The evolution of gonadotropins : some molecular data concerning a non-mammalian pituitary gonadotropin, the hormone from a teleost fish (Cyprinus carpio L.). Biochimie, 59, 893-898.

KERKOF P. R., BOSCHWITZ D., GORBMAN A., 1973. The response of hagfish thyroid tissue to thyroid inhibitors and to mammalian thyroid stimulating hormone. Gen. comp. Endocrinol., 21, 231-240.

LAFAURIE M., FORMENTO J. L., KREBS B. P., 1978. Détermination des stades caraçéristiques du cycle biologique de Mullus barbatus Linné. Dosages radioimmunologiques des hormones thyroïdiennes. IVe journ. Etud. Pollutions Antalya, C. I. E. S. M., 325-329.

LAM T. J., PANDEY S., NAGAHAMA Y., HOAR W. S., 1978. Endocrine control of oogenesis, ovulation and oviposition in goldfish, 55-64. In GAILLARD P. J., BOER H. H., Comparative Endocrinology, Elsevier/North Holland, Biomed. Press, Amsterdam.

LAMBERT J. G. D., 1978. Steroidogenesis in the ovary of Brachydanio rerio (Teleostei), 65-68. In GAILLARD P. J., BOER H. H., Comparative Endocrinology, Elsevier/North Holland, Biomed. Press, Amsterdam.

LANCE V., CALLARD I. P., 1978. In vivo responses of female snakes (Natrix fasciata) and female turtles (Chrysemis picta) to ovine gonadotropins (FSH and LH) as measured by plasma progesterone, testosterone and estradiol levels. Gen. comp. Endocrinol., 35, 295-301.

LARSEN L. O., 1973. Development in adult, freshwater river lampreys and its hormonal control ; starvafion, sexual maturation and natural death, 1-172, Thes., Copenhagen.

LICHT P., 1978. Studies on the immunochemical relatedness among Tetrapod gonadotropins and their subunits with antisera to sea turile hormones. Gen. comp. Endocrinol., 36, 68-78.

LICHT P., 1979. Reproductive endocrinology of Reptiles and Amphibians : gonadotropins. Annu. Rev. Physiol., 41, 337-353.

LICHT P., BONA-GALLO A., 1978. Immunochemical relatedness among pituitary follicle stimulating hormones of tetrapod Vertebrates. Gen. comp. Endocrinol., 36, 575-584.

LICHT P., MIDGLEY A. R. Jr, 1976. Competition for the in vitro binding of radioiodinated human follicle-stimulating hormone in reptilian, avian and mammalian gonads by non mammalian gonadotropins. Gen. comp. Endocrinol., 30, 364-371.

LICHT P., PAPKOFF H., FARMER S. W., MULLER C. H., TSUI H. W., CREWS D., 1977. Evolution of gonadotropin structure and function Rec. Progr., Horm. Res., 33, 169-248.

LICHT P., FARMER S. W., PAPKOFF H., 1978. Biological activity of hybrid combinations of ovine and sea turtle LH subunits. Gen. comp. Endocrinol., 35, 289-294.

LINDSEY A. M., CHANNING C. P., 1979. Comparison of the stimulatory effects of ovine, porcine and human follicle stimulating hormone and of ovine and human luteinizing hormone on the accumulation of cyclic AMP by porcine granulosa cells. J. Endocrinol., 80, 9-20.

MC KENZIE D. S., LICHT P., PAPKOFF H., 1978. Thyrotropin from Amphibian (Rana cafesbeiana) pituitaries and evidence for heterothyrotropic activity of bullfrog luteinizing hormone in Reptiles. Gen. comp. Endocrinol., 36, 566-574. 
NAYYAR S. K., KESHAVANATH P., SUNDARARAJ B. 1., DONALDSON E. M., 1976. Maintenance of spermatogenesis and seminal vesicles in the hypophysectomized catfish, Heteropneustes fossilis (Bloch) : effects of ovine and salmon gonadotropin, and testosterone. Can. J. Zool., 54, 285-292.

NG T. B., IDLER D. R., 1978a. A vitellogenic hormone with a large and a small form from salmon pituitaries. Gen. comp. Endocrinol., 35, 189-195.

NG T. B., IDLER D. R., 1978b. "Big » and " little » forms of plaice vitellogenic and maturational hormones. Gen. comp. Endocrinol., 34, 408-420.

OHNO S., 1970. Evolution by gene duplication, 1-160. Springer-Verlag.

OLIVEREAU M., CHAMBOLLE P., 1978. Ultrastructure des cellules gonadotropes de l'anguille normale et après injection d'œstradiol. C. R. Acad. Sci. Paris, sér. D, 287, 1409-1412.

ORGEL L. E., 1977. Gene duplication and the origin of proteins with novel functions. J. theor Biol., 67773.

PAPKOFF H., FARMER S. W., LICHT P., 1976. Isolation and characterization of follicle stimulating hormone and luteinizing hormone and its subunits from snapping turtle (Chelydra serpentina) pituitaries. Endocrinology, 98, 767-777.

PARLOW A. F., 1961. Bioassay of pituitary luteinizing hormone by depletion of ovarian ascorbic acid, 300-310. In ALBERT A., Human pituitary gonadotropins, Ch. Thomas Publ.

PETER R. E., 1978. Regulating mechanisms of the anterior pituitary, 341-352. In GAILLARD P. J., BOER H. H., Comparative Endocrinology, Elsevier/North Holland Biomed. Press, Amsterdam.

MARCHELIDON J., FONTAINE-BERTRAND E., SALESSE R., GARNIER J., BURZAWA-GÉRARD E., FONTAINE Y. A., 1978. Rapidité de la réassociation in vitro des sous-unités de gonadotropine chez un poisson Téléostéen, la carpe (Cyprinus carpio L.). C. R. Acad. Sci. Paris, sér. D, 287, 301-304.

MARCHELIDON J., SALESSE R., GARNIER J., BURZAWA-GÉRARD E., FONTAINE Y. A., 1979. Fish versus mammal gonadotropins : kinetics of subunits association depend on the zoological origin., Nafure, 281, 314-315.

MATTY A. J., TSUNEKI K., DICKHOFF W. W., GORBMAN A., 1976. Thyroid and hgonadal function in hypophysectomized hagfish, Eptatretus stouti. Gen. comp. Endocrinol., 30, 500-516.

MAUNG Z. W., FOLLETT B. K., 1977. Effects of chicken and ovine luteinizing hormone on androgen release and cyclic AMP production by isolated cells from the quail testis. Gen. comp. Endocrinol., 33, 242-253.

MEANS A. R., FAKUNDING J. L., HUCKINS C., TINDALL D. J., VITALE R., 1976. Follicle stimulating hormone, the Sertoli cell and spermatogenesis. Rec. Progr. Horm. Res., 32, 477-527.

MULLER C. H., 1977. Plasma 5 $\alpha$-dihydrotestosterone and festosterone in the bullfrog, Rana catesbeiana stimulation by bullfrog LH. Gen. comp. Endocrinol., 33, 122-133.

MULNER O., THIBIER C., OZON R., 1978. Steroid biosynthesis by ovarian follicles of Xenopus laevis in vitro during oogenesis. Gen. comp. Endocrinol., 34, 287-295.

NATH P., SUNDARARAJ B. I., 1978. Endocrine control of vitellogenesis in the catfish Heferopneuste fossilis, 90. In GAILLARD P. J. BOER H. H., Comparative Endocrinology, Elsevier/North Holland Biomed. Press, Amsterdam.

PIERCE J. G., LIAO T. H., HOWARD S. M., SHOME B., CORNELL J. S., 1971 . Studies on the structure of thyrotropin and its relationship to luteinizing hormone. Rec. Progr. Horm. Res., 27, 165-212.

PETER R. E., CRIM L. W., 1979. Reproductive endocrinology of fishes : gonadal cycles and gonadotropin in Teleosts. Annu. Rev. Physiol., 41, 323-337.

PICKERING A. D., 1972. Effects of hypophysectomy on the activity of the endostyle and thyroid gland in the larval and adult river lamprey, Lampetra fluviatilis L. Gen. comp. Endocrinol., 18, 335-343.

PIERCE J. G., FAITH M. R., DONALDSON E. M., 1976. Antibodies to reduced S-carboxymethylated alpha-subunit of bovine luteinizing hormone and their application to study of the purification of gonadotropin from salmon (Oncorhynchus tshawytscha) pituitary glands. Gen. comp. Endocrinol., 30, 47-61.

RICE B. F., 1978. Stéroïdogenèse dans l'ovaire humain. Aspect compartimental. Acquisitions récentes, 55-76. In SCHOLLER R., Endocrinologie de l'ovaire, SEPE, Paris. 
SALMON C., DELERUE-LE BELLE N., FONTAINE Y. A., 1974a. La stimulation hormonale de l'adényl cyclase ovarienne chez le rat et chez un poisson téléostéen (Carassius auratus) : influence de la concentration en adénosine triphosphate. C. R. Acad. Sci. Paris, sér. D, 278, 1959-1962.

SALMON C., DELERUE-LE BELLE N., FONTAINE Y. A., 1974b. Influence de la température sur l'adényl cyclase ovarienne et sa stimulation hormonale. Ełude comparée chez le rat et un poisson téléostéen (Carassius auratus). Biochimie, 56, 1229-1238.

SALMON C., DELERUE-LE BELLE N., FONTAINE Y. A., 1980. Adénylałe cyclase de l'ovaire et de la thyroïde de Mammifère : interactions des hormones (LH, FSH, TSH) mammaliennes avec une gonadotropine de poisson téléostéen et ses sous-unités. Soumis à Gen. comp. Endocrinol.

SAXENA B. B., RATHNAM P., 1978. The structure and function of follicle-stimulating hormone, 183212. In MC KERNS K. W., Structure and function of the gonadotropins, Plenum Press, New York, and London.

SCANES C. G., FOLLETT B. K., GOOS H. J. Th., 1972. Cross reaction in a chicken LH radioimmunoassay with plasma and pituitary extracts from various species. Gen. comp. Endocrinol., 19, 596-600.

SHAHABI N. A., BAHR J. M., NALBANDOV A. V., 1975. Effect of $L H$ injection on plasma and follicular steroids in the chicken. Endocrinology, 96, 969-972.

STEELMAN S. L., POHLEY F. M., 1953. Assay of the follicle stimulating hormone based on the augmentation with human chorionic gonadotropin. Endocrinology, 53, 604-616.

STEHLE B., 1978. Stimulation of androgen production in male immature Xenopus laevis by mammalian gonadotropins, 86. In GAILLARD P. J., BOER H. H., Comparative Endocrinology, Elsevier/ North Holland Biomed. Press, Amsterdam.

STEHLE-KAHL B., SCHULTHEISS H., 1980. Gonadotropic and thyrotropic activities of gel electrophoretically separated Xenopus pituitary proteins in Xenopus laevis D. Gen. comp. Endocrinol. (sous presse).

SUMPTER J. P., FOLLETT B. H., JENKINS N., DODD J. M., 1978a. Studies on the purification and properties of gonadotrophin from ventral lobes of the pituitary gland of the dogfish (Scyliorhinus canicula). Gen. comp. Endocrinol., 36, 264-274.

SUMPTER J. P., JENKINS N., DODD J. M., 1978b. Gonadotrophic hormone in the pituitary gland of the dogfish (Scyliorhinus canicula) : distribution and physiological significance. Gen. comp. Endocrinol., 36, 275-285.

SUNDARARAJ B. I., ANAND T. C., 1972. Effects of piscine and mammalian gonadotropins on gametogenesis in the catfish, Heteropneustes fossilis. Gen. comp. Endocrinol., suppl. 3, 688702.

SUNDARARAJ B. I., GOSWAMI S. V., 1977. Hormonal regulation of in vivo and in vitro oocyte maturation in the catfish, Heferopneuste fossilis. Gen. comp. Endocrinol., 32, 17-28.

SUNDARARAJ B. I., NAYYAR S. K., BURZAWA-GÉRARD E., FONTAINE Y. A., 1976. Effects of carp gonadotropin on ovarian maintenance, maturation and ovulation in hypophysectomized catfish, Heteropneustes fossilis. Gen. comp. Endocrinol., 30, 472-476.

SUNDARARAJ B. I., NATH P., JEET V., 1978. Role of circadian and circannual rhythms in the regulation of ovarian cycles in fishes : a catfish model, 137-140. In GAILLARD P. J., BOER H.H., Comparative Endocrinology, Elsevier/North Holland Biomed. Press, Amsterdam.

TALIADOUROS G. S., CANFIELD R. E., NISULA B. C., 1978. Thyroid-stimulating activity of chorionic gonadotropin and luteinizing hormone. J. clin. Endoc. Metab., 47, 855-860.

TAN E. S. P., DODD J. M., 1978. Immunological rrelatedness of gonadotrophins of various fishes as shown by radioimmunoassays. Ann. Biol. anim. Bioch. Biophys., 18, 759-1106.

TANG F., CHAN S. T. H., LOFTS B., 1974. Effect of mammalian luteinizing hormone on the natural sex reversal of the Rice-Field eel, Monopterus albus (Zuiew). Gen. comp. Endocrinol., 24, 242-248.

THIBAULT C., 1977. Are follicular maturation and oocyte maturation independent processes ? J. Reprod. Fert., 51, 1-15.

TOKARZ R. R., 1978. An autoradiographic study of the effects of mammalian gonadotropins (follicle stimulating hormone and luteinizing hormone) and estradiol $17 \beta$ on ${ }^{3} \mathrm{H}$ thymidine labeling of surface epithelial cells, prefollicular cells and oogonia in the ovary of the lizard Anolis carolinensis. Gen. comp. Endocrinol., 35, 179-188. 
TSAFRIRI A., LINDNER H. R., 1978. Endocrine control of oocyle maturation in cultured graafian follicles of the rat, 77-80. In GAILLARD P. J., BOER H. H., Comparative Endocrinology, Elsevier/ North Holland Biomed. Press, Amsterdam.

TSUTSUI K., ISHII S., 1978. Effects of follicle-stimulating hormone and testosterone on receptors of follicle-stimulating hormone in the testis of the immature Japanese quail. Gen. comp. Endocrinol., 36, 297-305.

UPADHYAY S. N., BRETON B., BILLARD R., 1978. Ultrastructural studies on experimentally induced vitellogenesis in juvenile rainbow trout (Salmo gairdneri R.). Ann. Biol. anim. Bioch. Biophys., 18, 1019-1025.

VAITUKAITIS J. L., ROSS G. T., BRAUNSTEIN G. D., RAYFORD P. L., 1976. Gonadotropins and their subunits : basic and clinical studies. Rec. Progr. Horm. Res., 32, 289-331.

VAN OORDT P. G. W. J., EKENGREEN B., 1978. The gonadotropic cell in the pituitary of Teleosts and the central regulation of its activity, 353-356. In GAILLARD P. J., BOER H. H., Comparative Endocrinology, Elsevier/North Holland Biomed. Press, Amsterdam.

YAMAZAKI F., 1965. Endocrinological studies on the reproduction of the female goldfish, Corossius auratus L., with special reference to the function of the pituitary gland. Mem. Fac. Fish., Hokkaido Univ., 13, 1-64.

YAMAZAKI F., DONALDSON E. M., 1968. The effects of partially purified salmon pituitary gonadotropin on spermatogenesis, vitellogenesis and ovulation in hypophysectomized goldfish, Carassius auratus. Gen. comp. Endocrinol., 11, 292-299.

ZELEZNIK A. J., KEYES P. L., MENON K. M. J., MIDGLEY A. R., REICHERT R. E., 1977. Development-dependent responses of ovarian follicles to FSH and h-CG. Amer. J. Physiol., 233, E 229E 234.

ZUCKERKANDL E., 1975. The appearance of new structures and functions in proteins during evolution. J. molec. Evol., 7, 1-57. 